ESAIM: M2AN 47 (2013) 609-633

DOI: $10.1051 / \mathrm{m} 2 \mathrm{an} / 2012042$
ESAIM: Mathematical Modelling and Numerical Analysis

www.esaim-m2an.org

\title{
ANALYSIS OF AN ASYMPTOTIC PRESERVING SCHEME FOR RELAXATION SYSTEMS *
}

\author{
Francis Filbet ${ }^{1}$ AND AmÉLie RAmbaud ${ }^{1}$
}

\begin{abstract}
We consider an asymptotic preserving numerical scheme initially proposed by F. Filbet and S. Jin [J. Comput. Phys. 229 (2010)] and G. Dimarco and L. Pareschi [SIAM J. Numer. Anal. 49 (2011) 2057-2077] in the context of nonlinear and stiff kinetic equations. Here, we propose a convergence analysis of such a scheme for the approximation of a system of transport equations with a nonlinear source term, for which the asymptotic limit is given by a conservation law. We investigate the convergence of the approximate solution $\left(u_{h}^{\varepsilon}, v_{h}^{\varepsilon}\right)$ to a nonlinear relaxation system, where $\varepsilon>0$ is a physical parameter and $h$ represents the discretization parameter. Uniform convergence with respect to $\varepsilon$ and $h$ is proved and error estimates are also obtained. Finally, several numerical tests are performed to illustrate the accuracy and efficiency of such a scheme.
\end{abstract}

Mathematics Subject Classification. 35L02, 82C70, 65M06.

Received January 5, 2012. Revised August 7, 2012

Published online January 15, 2013.

\section{INTRODUCTION}

The numerical resolution of kinetic and hyperbolic equations is a challenging task because these models often contain small parameters describing multiscale phenomena. It is particularly difficult to capture the behavior of the solution characterized by time multiscale features since a straightforward discretization leads typically to stiff differential system of equations. Such problems are encountered in many physical applications, for example rarefied gas dynamics $[9,10]$, semiconductor modeling [8], quasi-neutral plasma simulations [7], the list of possible applications being not exhaustive. The motivation of this work is closely related to rarefied gas dynamics simulations [10]. In this case the source term is represented by the nonlinear and non local Boltzmann operator modelling collision mechanism of particles, the intensity of collisions $1 / \varepsilon$ reaching several orders of magnitude. The difficulty with these stiff problems is that they become singular in the limit $\varepsilon \rightarrow 0$, where $\varepsilon$ is a small parameter responsible of the stiffness. A straightforward discretization of such problems (using, for example, explicit Runge-Kutta schemes) generates a very restrictive condition on the time step $\Delta t=O(\varepsilon)$, which becomes unfeasible for $\varepsilon \ll 1$. In this paper we present a new approach based on the so called Asymptotic

Keywords and phrases. Hyperbolic equations with relaxation, fluid dynamic limit, asymptotic-preserving schemes.

* Both authors are partially supported by the European Research Council ERC Starting Grant 2009, project 239983-NuSiKiMo.

1 Université de Lyon, UMR5208, Institut Camille Jordan, Université Claude Bernard Lyon 143 boulevard 11 novembre 1918 , 69622 Villeurbanne Cedex, France. filbet@math.univ-lyon1.fr; rambaud@math.univ-lyon1.fr 
Preserving reformulation introduced initially in [16]. This work presents an important improvement in the rigorous convergence analysis of an asymptotic preserving scheme proposed in $[9,10]$ in the framework of kinetic equations with stiff operators. Of course, a complete analysis seems to be tricky for fully nonlinear kinetic equations like the Boltzmann equation. Therefore, we focus on a simple hyperbolic relaxation system, which is the standard benchmark model for all relaxation problems : for all $(t, x)$ in $\mathbb{R}^{+} \times \mathbb{T}$ :

$$
\left\{\begin{array}{l}
\partial_{t} u^{\varepsilon}+\partial_{x} v^{\varepsilon}=0, \quad u^{\varepsilon}(0, x)=u_{0}^{\varepsilon}(x) \\
\partial_{t} v^{\varepsilon}+a \partial_{x} u^{\varepsilon}=-\frac{1}{\varepsilon} \mathcal{R}\left(u^{\varepsilon}, v^{\varepsilon}\right), \quad v^{\varepsilon}(0, x)=v_{0}^{\varepsilon}(x),
\end{array}\right.
$$

where $a>0$ is a constant coefficient to be discussed later, $\varepsilon$ is the relaxation parameter, which can be either large or small (leading to a stiff source term) and $\mathcal{R}: \mathbb{R} \times \mathbb{R} \mapsto \mathbb{R}$ is a nonlinear function such that $\mathcal{R}(0,0)=0$. The system of equations (1.1) is often referred as a two velocity kinetic model and it has been intensively studied for many years [3]. Here we will assume that the function $\mathcal{R} \in \mathcal{C}^{1}(\mathbb{R} \times \mathbb{R}, \mathbb{R})$ and its partial derivative with respect to the second variable is bounded from below, then from the implicit function theorem it possesses a unique local equilibrium,

$$
\mathcal{R}(u, v)=0 \Leftrightarrow v=A(u),
$$

where $A$ is a locally Lipschitz continuous function with $A(0)=0$. Therefore, under some assumptions on $\mathcal{R}$ and on the initial data, the solution $\left(u^{\varepsilon}, v^{\varepsilon}\right)$ to (1.1) converges to $(u, v)$ with $v=A(u)$ and $u$ solution to the conservation law $[6,18]$

$$
\left\{\begin{array}{l}
\partial_{t} u+\partial_{x} A(u)=0, \text { in } \mathbb{R}^{+} \times \mathbb{T}, \\
u(t=0)=u_{0}
\end{array}\right.
$$

where the initial datum $u_{0}$ is given by

$$
u_{0}=\lim _{\varepsilon \rightarrow 0} u_{0}^{\varepsilon}
$$

Developing robust numerical schemes for (1.1) that work in various asymptotic regimes (for a wide range of values of $\varepsilon>0$ ) becomes challenging. It has been done in the framework of Asymptotic-Preserving (AP) schemes $[10,16]$. From a numerical point of view, the treatment of the stiffness cannot be done with explicit schemes, so that semi-implicit or fully implicit schemes have to be applied, but this procedure becomes time consuming and should be avoided when the operator $\mathcal{R}$ is nonlinear and non local since it requires an additional step for the numerical resolution of a nonlinear problem. One solution offered by E. Gabetta, L. Pareschi and G. Toscani [12] to design an uniformly stable scheme, was to penalize the nonlinear collision operator $\mathcal{R}$ by a linear function $\beta v$ and then absorb the linearly stiff part into the time variable to remove the stiffness.

More recently, F. Filbet and S. Jin [10] proposed to penalize the operator $\mathcal{R}$ by the BGK type operator $\beta(A(u)-v)$ in order to build stable schemes with respect to $\varepsilon>0$. If such schemes are now numerically validated and extensively used to discretize various equations $[9-12,16]$, their mathematical study has only been done in some particular cases [13,14]. Indeed, few works are devoted to the mathematical analysis of asymptotic preserving schemes for the approximation of hyperbolic systems with source terms. Actually most of the theoretical works concern the numerical analysis of Relaxation scheme where (1.1) is only solved in the asymptotic limit $\varepsilon \rightarrow 0$ and for simple source terms. We refer for instance to the series of paper $[1,2]$ and then $[5,17,19,23]$ on relaxation schemes. The requirement of asymptotic preserving scheme is to be uniformly accurate and stable for all range values of $\varepsilon>0$.

Therefore, the goal of the present paper mainly consists in providing error estimates between the approximate solution $\left(u_{h}^{\varepsilon}, v_{h}^{\varepsilon}\right)$ and the exact solution to (1.1) which are uniform with respect to $\varepsilon$ and the numerical parameter $h$. Here we propose a rigorous analysis of the Asymptotic Preserving scheme proposed by F. Filbet and S. Jin [10] and G. Dimarco and L. Pareschi [9] for a nonlinear relaxation operator. 
Concerning the mathematical study of the system (1.1), we refer for instance to [20] for convergence proof and to $[21,22]$ for error estimates.

Theorem 1.1. Assume that the initial datum $\left(u_{0}^{\varepsilon}, v_{0}^{\varepsilon}\right)$ is bounded independently of $\varepsilon$ in $B V(\mathbb{T})$. Consider $\mathcal{R} \in$ $\mathcal{C}^{1}(\mathbb{R} \times \mathbb{R}, \mathbb{R})$, with $\mathcal{R}(0,0)=0$ and $\nabla \mathcal{R}$ is uniformly bounded with respect to $v$ and locally bounded with respect to $u$ such that, for any $(u, v)$ in $\left[-U_{0}, U_{0}\right] \times \mathbb{R}$ :

$$
\left\{\begin{array}{l}
\left|\partial_{u} \mathcal{R}(u, v)\right| \leq g\left(U_{0}\right), \\
0<\beta_{0}\left(U_{0}\right) \leq \partial_{v} \mathcal{R}(u, v) \leq h\left(U_{0}\right),
\end{array}\right.
$$

where $\beta_{0}, g$ and $h$ are some constants depending only on $U_{0}$. Then (1.2) is satisfied and there exists a characteristic speed $a>0$ large enough (see the condition (2.6) below) such that the system (1.1) admits a unique solution $\left(u^{\varepsilon}, v^{\varepsilon}\right)$ in $\mathcal{C}\left(\left[0, \infty\left[, L^{1}(\mathbb{T})^{2}\right)\right.\right.$ and there exists a constant $C>0$ which only depends on a and $\left(u_{0}^{\varepsilon}, v_{0}^{\varepsilon}\right)$, such that for any $\varepsilon>0$ :

$$
\left\{\begin{array}{l}
\left\|v^{\varepsilon}(t) \pm \sqrt{a} u^{\varepsilon}(t)\right\|_{\infty}+T V\left(u^{\varepsilon}(t)\right)+T V\left(v^{\varepsilon}(t)\right) \leq C \quad \forall t>0 \\
\left\|u^{\varepsilon}(t+\tau)-u^{\varepsilon}(t)\right\|_{1} \leq C \tau, \quad \forall t \in \mathbb{R}^{+}, \tau \in \mathbb{R}^{+}, \\
\left\|v^{\varepsilon}(t+\tau)-v^{\varepsilon}(t)\right\|_{1} \leq \frac{C}{\varepsilon} \tau, \quad \forall t \in \mathbb{R}^{+}, \tau \in \mathbb{R}^{+}, \\
\left\|v^{\varepsilon}(t+\tau)-v^{\varepsilon}(t)\right\|_{1} \leq C_{\nu} \tau, \quad \forall t \geq \nu, \tau \in \mathbb{R}^{+}
\end{array}\right.
$$

where $\nu>0$, and $C_{\nu}$ only depends on $a,\left(u_{0}^{\varepsilon}, v_{0}^{\varepsilon}\right)$ and $\nu$. Moreover, there exists $\beta_{0}>0$ such that,

$$
\left\|v^{\varepsilon}(t, \cdot)-A\left(u^{\varepsilon}(t, \cdot)\right)\right\|_{1} \leq C\left[\mathrm{e}^{-\frac{\beta_{0} t}{\varepsilon}}\left\|v_{0}^{\varepsilon}-A\left(u_{0}^{\varepsilon}\right)\right\|_{1}+\varepsilon\right] .
$$

Finally, if $u_{0}^{\varepsilon}$ converges, as $\varepsilon$ goes to zero, to $u_{0}$ defined by (1.4), then the sequence $\left(u^{\varepsilon}, v^{\varepsilon}\right)$ converges to $(u, A(u))$ when $\varepsilon$ goes to 0 , such that, for any $\nu>0$ : for all $T>0$,

$$
\left\|u^{\varepsilon}(t)-u(t)\right\|_{L^{1}}+\left\|v^{\varepsilon}(t)-A(u(t))\right\|_{L^{1}} \leq C_{t} \sqrt{\varepsilon}, \quad \nu \leq t \leq T,
$$

where $C_{T}>0$ depends on the initial data and $T$ and $u$ is the unique entropic solution to the Cauchy problem (1.3).

The paper is organized as follows. We present in Section 2 the asymptotic preserving scheme for the relaxation model, and state the both convergence results of the Asymptotic Preserving scheme when the relaxation parameter $\varepsilon$ goes to zero (Thm. 2.2) and next when the discretization parameter $h$ goes to zero (Thm. 2.3). Then, we establish some a priori estimates in $L^{\infty}$ and $B V$ on the numerical solution to the Asymptotic Preserving scheme in Section 3 in order to prove both the zero relaxation limit (Sect. 4) and the convergence of the scheme (Sect. 5). Notice that these a priori estimates are uniform both with respect to the relaxation parameter $\varepsilon$ and the time step $\Delta t$ and space step $\Delta x$. Finally, we present some numerical results in Section 6 .

\section{NumericAl SCHEMES AND MAIN RESUlts}

We remind that when $\mathcal{R}(u, v)=v-A(u)$, where $A \in \mathcal{C}^{1}(\mathbb{R}, \mathbb{R})$ is a given function, the necessary and sufficient stability condition is given by the so called sub-characteristic condition $[3,16]$ :

$$
\left|A^{\prime}(u)\right|<\sqrt{a} .
$$


It means that the propagation speed of the equilibrium problem has to be bounded by the speeds of the relaxation system, which is therefore dissipative. For more details about this case, we refer to $[6,18,20]$.

Hence the sub-characteristic condition reads, in our case:

$$
\left|\frac{\partial_{u} \mathcal{R}(u, v)}{\partial_{v} \mathcal{R}(u, v)}\right|<\sqrt{a}
$$

For the sequel, we define for any $N>0$ and $\alpha>0$,

$$
\left\{\begin{array}{l}
U(N, \alpha):=\left(1+\frac{1}{\sqrt{\alpha}}\right) N, \\
F(N, \alpha):=\sup _{|\xi| \leq U(N, \alpha)}|A(\xi)|, \\
V(N, \alpha):=U(N, \alpha)+\frac{1}{\sqrt{\alpha}} F(N, \alpha) .
\end{array}\right.
$$

We also denote by $I(N, \alpha)$ the compact set

$$
I(N, \alpha):=[-\sqrt{a} V(N, \alpha), \sqrt{a} V(N, \alpha)]^{2} .
$$

Moreover, we assume that the initial conditions $u_{0}^{\varepsilon}, v_{0}^{\varepsilon}$ are bounded independently of $\varepsilon$ in $L^{\infty}(\mathbb{T})$, such that:

$$
N_{0}:=\max \left\{\sup _{\varepsilon>0}\left\|u_{0}^{\varepsilon}\right\|_{\infty}, \sup _{\varepsilon>0}\left\|v_{0}^{\varepsilon}\right\|_{\infty}\right\}<\infty .
$$

Consider any $a_{0}>0$ and assume that the function $\mathcal{R} \in \mathcal{C}^{1}(\mathbb{R} \times \mathbb{R}, \mathbb{R})$ satisfies (1.2) and (1.5). We choose the characteristic speed $\sqrt{a}>0$ and the parameter $\beta>0$ such that

$$
\sqrt{a}>\max \left\{\sqrt{a_{0}}, \frac{g\left(V\left(N_{0}, a_{0}\right)\right)}{\beta_{0}\left(V\left(N_{0}, a_{0}\right)\right)}\right\} \quad \text { and } \beta=h\left(V\left(N_{0}, a_{0}\right)\right),
$$

where $V$ is given by (2.3).

We present here the splitting Asymptotic Preserving scheme and its relaxed version. To this aim, we introduce a space time discretization based on a uniform grid of points $\left(x_{j+1 / 2}\right)_{j \in \mathbb{Z}} \subset \mathbb{T}$, with space step $\Delta x$, and discrete time $t^{n}=n \Delta t, n \in \mathbb{N}$, for which the time step $\Delta t$ satisfies the CFL condition:

$$
0<\lambda:=\frac{\sqrt{a} \Delta t}{\Delta x}<1 .
$$

We denote by $h=(\Delta t, \Delta x)$ the discretization parameter.

\subsection{An Asymptotic Preserving scheme for the relaxation system}

In this section, we design a numerical scheme for system (1.1), by introducing a splitting between the linear transport part, and the nonlinear relaxation part, for which we will take advantage of the knowledge of the equilibrium (1.2). In the asymptotic regime $\varepsilon \rightarrow 0$, the second equation of (1.1) becomes stiff and explicit schemes are subject to a stability constraint $\Delta t=O(\varepsilon)$. Of course, implicit schemes allow larger time steps, but new difficulty arises in computing the numerical solution of a fully nonlinear problem at each time step. Here we want to combine both advantages of implicit and explicit schemes, that is, large time step for stiff problems and low computational complexity of the numerical solution at each time step. This is done, as said in the introduction, in the spirit of Asymptotic Preserving schemes for nonlinear relaxation problems introduced by F. Filbet and S. Jin [10] and G. Dimarco and L. Pareschi [9]. 
Thus we construct a numerical solution $\left(u_{h}^{\varepsilon}, v_{h}^{\varepsilon}\right)$ to $(1.1)$ for $(t, x) \in \mathbb{R}^{+} \times \mathbb{T}$ as follows

$$
\left\{\begin{array}{l}
u_{h}^{\varepsilon}(t, x)=\sum_{n \in \mathbb{N}} \sum_{j \in \mathbb{Z}} u_{j}^{n} \mathbf{1}_{C_{j}}(x) \mathbf{1}_{\left[t^{n}, t^{n+1}[\right.}(t), \\
v_{h}^{\varepsilon}(t, x)=\sum_{n \in \mathbb{N}} \sum_{j \in \mathbb{Z}} v_{j}^{n} \mathbf{1}_{C_{j}}(x) \mathbf{1}_{\left[t^{n}, t^{n+1}[\right.}(t)
\end{array}\right.
$$

where $\left.C_{j}=\right] x_{j-1 / 2}, x_{j+1 / 2}\left[\right.$ are the space cells and the sequences $\left(u_{j}^{n}\right)_{n, j}$ and $\left(v_{j}^{n}\right)_{n, j}$ depend on $\varepsilon$ and are given below.

First, initial data are computed as the averaged values of the initial data through each space cell: for all $j$ in $\mathbb{Z}$,

$$
u_{j}^{0}=\frac{1}{\Delta x} \int_{C_{j}} u_{0}^{\varepsilon}(x) \mathrm{d} x \quad \text { and } \quad v_{j}^{0}=\frac{1}{\Delta x} \int_{C_{j}} v_{0}^{\varepsilon}(x) \mathrm{d} x .
$$

Then, in order to discretize the system (1.1), we apply a splitting strategy into a linear transport part and a stiff ordinary differential part as follows. The first part consists to apply a time explicit scheme combined with a finite volume method to the following linear differential system

$$
\left\{\begin{array}{l}
\partial_{t} u+\partial_{x} v=0 \\
\partial_{t} v+a \partial_{x} u=0
\end{array}\right.
$$

and then the second part deals with the stiff ordinary differential equations

$$
\left\{\begin{array}{l}
\partial_{t} u=0 \\
\partial_{t} v=-\frac{1}{\varepsilon} \mathcal{R}(u, v) .
\end{array}\right.
$$

We first approximate the linear transport part (2.10), that is, for a given $\left(u^{n}, v^{n}\right)$, we compute $\left(u^{n+1 / 2}, v^{n+1 / 2}\right)$ at time $t^{n+1}$ with a standard Finite Volume scheme, that is, for all $j \in \mathbb{Z}$,

$$
\left\{\begin{array}{l}
u_{j}^{n+1 / 2}=u_{j}^{n}-\Delta t D_{h} v_{j}^{n}, \\
v_{j}^{n+1 / 2}=v_{j}^{n}-\Delta t a D_{h} u_{j}^{n},
\end{array}\right.
$$

where $D_{h} v_{j}^{n}$ and $a D_{h} u_{j}^{n}$ are discrete derivatives with respect to $x$ of $v$ and $u$, given for instance by a Lax-Friedrichs scheme, namely:

$$
\left\{\begin{array}{l}
D_{h} v_{j}^{n}=\frac{1}{2 \Delta x}\left[\left(v_{j+1}^{n}-v_{j-1}^{n}\right)-\sqrt{a}\left(u_{j+1}^{n}-2 u_{j}^{n}+u_{j-1}^{n}\right)\right], \\
a D_{h} u_{j}^{n}=\frac{1}{2 \Delta x}\left[a\left(u_{j+1}^{n}-u_{j-1}^{n}\right)-\sqrt{a}\left(v_{j+1}^{n}-2 v_{j}^{n}+v_{j-1}^{n}\right)\right] .
\end{array}\right.
$$

Remark 2.1. Of course, there is a wide range of possible choices for the numerical fluxes. As we will see below, the main property of the numerical scheme for the linear transport term that we require is the TVD (Total Variation Diminishing) property, namely, for all $n \in \mathbb{N}$,

$$
T V\left(u^{n+1 / 2}\right) \leq T V\left(u^{n}\right) \text { and } T V\left(v^{n+1 / 2}\right) \leq T V\left(v^{n}\right),
$$

where $T V(u):=\sum_{j \in \mathbb{Z}}\left|u_{j+1}-u_{j}\right|$. 
Hence, the second part of the splitting only consists in approximating the nonlinear ordinary differential equation (2.11), for all $j \in \mathbb{Z}$, starting from $\left(u_{j}^{n+1 / 2}, v_{j}^{n+1 / 2}\right)$. To this aim, we use the decomposition

$$
\mathcal{R}(u, v)=[\mathcal{R}(u, v)-\beta(v-A(u))]+\beta(v-A(u)),
$$

where $\beta>0$ is given by (2.6). Then, we apply a time exponential scheme on the dissipative part and get the following numerical scheme:

$$
\left\{\begin{aligned}
u_{j}^{n+1}= & u_{j}^{n+1 / 2}, \\
v_{j}^{n+1}= & v_{j}^{n+1 / 2}-\left(v_{j}^{n+1 / 2}-A\left(u_{j}^{n+1 / 2}\right)\right)\left[1-\left(1+\frac{\beta \Delta t}{\varepsilon}\right) \mathrm{e}^{-\beta \Delta t / \varepsilon}\right] \\
& -\frac{\Delta t}{\varepsilon} \mathrm{e}^{-\beta \Delta t / \varepsilon} \mathcal{R}\left(u_{j}^{n+1 / 2}, v_{j}^{n+1 / 2}\right) .
\end{aligned}\right.
$$

This numerical scheme (2.12)-(2.14) allows to define the sequence $\left(u_{j}^{n}, v_{j}^{n}\right)_{(j, n) \in \mathbb{Z} \times \mathbb{N}}$.

\subsection{Convergence results}

We first establish a convergence result on the asymptotic behavior of the numerical solution to (2.12)-(2.14) when $\varepsilon$ tends to zero and $h=(\Delta t, \Delta x)$ such that the CFL condition (2.7) is satisfied.

Theorem 2.2 (discrete asymptotic limit: $h$ fixed and $\varepsilon \rightarrow 0$ ). Under the assumption of Theorem 1.1 where $\mathcal{R}$ satisfies (1.2) and (1.5), $N_{0}$ is given by (2.5), the characteristic speed $\sqrt{a}>0$ and the parameter $\beta>0$ are given by (2.6). We also assume that the CFL condition (2.7) is verified. Then, the solution $\left(u_{h}^{\varepsilon}, v_{h}^{\varepsilon}\right)$ given by $(2.8)$ to the scheme (2.12)-(2.14) with the initial data (2.9), converges in $L^{1}(\mathbb{T})$, as $\varepsilon \rightarrow 0$, to a numerical solution $\left(u_{h}, v_{h}\right)$, that is,

$$
\left\|u_{h}^{\varepsilon}(t)-u_{h}(t)\right\|_{1}+\left\|v_{h}^{\varepsilon}(t)-v_{h}(t)\right\|_{1} \leq C_{t} \mathrm{e}^{-\beta_{0} \Delta t / \varepsilon}\left[1+\left\|\delta^{0}\right\|_{1}\right]+\left\|u_{h}^{\varepsilon}(0)-u_{h}(0)\right\|_{1}+\left\|v_{h}^{\varepsilon}(0)-v_{h}(0)\right\|_{1},
$$

where $C_{t}>0$ depends on the initial data and $t>0,\left(u_{h}, v_{h}\right)$ is a consistent approximation to the conservation laws (1.3) with $v_{h}=A\left(u_{h}\right)$,

$$
u_{h}(t, x):=\sum_{n \in \mathbb{N}} \sum_{j \in \mathbb{Z}} \bar{u}_{j}^{n} \mathbf{1}_{C_{j}}(x) \mathbf{1}_{\left[t^{n}, t^{n+1}[\right.}(t)
$$

and the sequence

$$
\bar{u}_{j}^{n+1}=\bar{u}_{j}^{n}-\Delta t D_{h} A\left(\bar{u}_{j}^{n}\right), \quad j \in \mathbb{Z}, n \geq 1,
$$

where the initial data $\bar{u}_{j}^{0}$ is the local average of $u_{0}$ is given by (1.4) and $\delta^{0}=u_{0}^{\varepsilon}-A\left(v_{0}^{\varepsilon}\right)$.

The proof of this theorem is analogous to the one corresponding to the continuous problem. The fact that we are able to establish uniform $B V$ bounds on the numerical solution allow to get error estimates with respect to $\varepsilon$.

On the other hand, we propose a convergence result of the Asymptotic Preserving scheme when $h=(\Delta t, \Delta x)$ goes to zero and $\varepsilon$ is fixed:

Theorem 2.3 (convergence analysis : $\varepsilon$ fixed and $h \rightarrow 0$ ). Under the assumption of Theorem 2.2, the solution $\left(u_{h}^{\varepsilon}, v_{h}^{\varepsilon}\right)$ to the scheme $(2.12)-(2.14)$ and the initial data (2.9) verifies for all $T>0$

$$
\left\|u_{h}^{\varepsilon}(t)-u^{\varepsilon}(t)\right\|_{1}+\left\|v_{h}^{\varepsilon}(t)-v^{\varepsilon}(t)\right\|_{1} \leq \frac{C_{t}}{\varepsilon}\left(\Delta t\left(\frac{\left\|\delta^{0}\right\|_{1}}{\varepsilon}+1\right)+\Delta x^{1 / 2}\right), \quad t \in[0, T] .
$$

where $C_{T}>0$ depends on the initial data and $T, \delta^{0}=u_{0}^{\varepsilon}-A\left(v_{0}^{\varepsilon}\right)$. 
Gathering these results together with Theorem 1.1, we get the following error estimates proving the uniform accuracy of the numerical scheme (2.12)-(2.14).

Theorem 2.4 (uniform error estimates with respect to $\varepsilon$ and $h$ ). Under the assumption of Theorem 2.2, we denote by

$$
\mathcal{E}_{h, \varepsilon}=\left\|u_{h}^{\varepsilon}(t)-u^{\varepsilon}(t)\right\|_{1}+\left\|v_{h}^{\varepsilon}(t)-v^{\varepsilon}(t)\right\|_{1},
$$

where $\left(u_{h}^{\varepsilon}, v_{h}^{\varepsilon}\right)$ is given by the scheme (2.12)-(2.14) and the initial data (2.9). Then we have for all $T>0$

$$
\mathcal{E}_{h, \varepsilon} \leq C_{T} \min \left(\frac{\Delta t}{\varepsilon}\left(\frac{\left\|\delta^{0}\right\|_{1}}{\varepsilon}+1\right)+\frac{\Delta x^{1 / 2}}{\varepsilon}, \mathrm{e}^{-\beta_{0} \Delta t / \varepsilon}\left[1+\left\|\delta^{0}\right\|_{1}\right]+\sqrt{\varepsilon}+\sqrt{\Delta x}\right), \quad \nu \leq t \leq T,
$$

where $C_{T}>0$ depends on the initial data and $T, \delta^{0}=u_{0}^{\varepsilon}-A\left(v_{0}^{\varepsilon}\right)$.

Proof. To prove this result we show that both error estimates are valid. On the one hand the classical convergence analysis in Theorem 2.3 gives for all $T>0$ that

$$
\mathcal{E}_{h, \varepsilon} \leq C_{T}\left(\frac{\Delta t}{\varepsilon}\left(\frac{\left\|\delta^{0}\right\|_{1}}{\varepsilon}+1\right)+\frac{\Delta x^{1 / 2}}{\varepsilon}\right), \quad \nu \leq t \leq T .
$$

On the other hand, we split $\mathcal{E}_{h, \varepsilon}$ as

$$
\mathcal{E}_{h, \varepsilon}=\mathcal{E}_{h, \varepsilon}^{1}+\mathcal{E}_{h}+\mathcal{E}_{\varepsilon}
$$

with the numerical error $\mathcal{E}_{h, \varepsilon}^{1}=\left\|u_{h}^{\varepsilon}(t)-u_{h}(t)\right\|_{1}+\left\|v_{h}^{\varepsilon}(t)-A\left(u_{h}(t)\right)\right\|_{1}$, where $\left(u_{h}, A\left(u_{h}\right)\right)$ corresponds to the numerical solution in the asymptotic limit $\varepsilon \rightarrow 0$ and is given by $(2.15)$, the term $\mathcal{E}_{h}=\left\|u(t)-u_{h}(t)\right\|_{1}+$ $\left\|v(t)-A\left(u_{h}(t)\right)\right\|_{1}$, where $(u, A(u)$ is the solution to the conservation laws (1.3) and the continuous error $\mathcal{E}_{\varepsilon}=\left\|u^{\varepsilon}(t)-u(t)\right\|_{1}+\left\|v^{\varepsilon}(t)-A(u(t))\right\|_{1}$. From Theorem 2.2, we have

$$
\mathcal{E}_{h}=\left\|u(t)-u_{h}(t)\right\|_{1}+\left\|v(t)-A\left(u_{h}(t)\right)\right\|_{1} \leq C_{T} \mathrm{e}^{-\beta_{0} \Delta t / \varepsilon}\left[1+\left\|\delta^{0}\right\|_{1}\right], \quad \nu \leq t \leq T .
$$

Then, the error $\mathcal{E}_{h}$ represents the $L^{1}$ error between the solution to the conservation laws (1.3) and its approximation by a first order Lax-Friedrichs scheme, which gives $\mathcal{E}_{h} \leq C_{T} \sqrt{\Delta x}$ and finally from Theorem 1.1, we have $\mathcal{E}_{\varepsilon} \leq C_{T} \sqrt{\varepsilon}$. Gathering these latter estimates, we get the second error estimate on $\mathcal{E}_{h, \varepsilon}$.

\section{A PRIORI ESTIMATES}

We first make sure that the sub-characteristic condition (2.2) is always satisfied to ensure the stability of the scheme (2.12)-(2.14) and prove estimates on the solution to the relaxation problem which are uniform with respect to $\varepsilon$. In following section, we drop the subscripts $\varepsilon$ for sake of clarity.

\subsection{A priori estimate on the relaxation operator}

Let us focus on the second part of the scheme devoted to the approximation of the relaxation source term and give a technical lemma, which establishes a quasi-monotonicity property on the operator $G_{\varepsilon, s}$ with $s>0$. In order to do this, we will rather consider the equivalent formulation on the diagonal variables $w$ and $z$. Let us rewrite the splitting scheme on these variables. For $u$ and $v$ given, we set

$$
w=-v-\sqrt{a} u \text { and } z=+v-\sqrt{a} u .
$$

Therefore, the linear transport scheme (2.12) written for $(w, z)$ exactly coincides with an upwind finite volume method: for all $j \in \mathbb{Z}$,

$$
\left\{\begin{array}{l}
w_{j}^{n+1 / 2}=w_{j}^{n}-\sqrt{a} \frac{\Delta t}{\Delta x}\left(w_{j}^{n}-w_{j-1}^{n}\right), \\
z_{j}^{n+1 / 2}=z_{j}^{n}+\sqrt{a} \frac{\Delta t}{\Delta x}\left(z_{j+1}^{n}-z_{j}^{n}\right),
\end{array}\right.
$$


whereas the nonlinear stiff part (2.14) yields, for all $j \in \mathbb{Z}$

$$
\left\{\begin{array}{l}
w_{j}^{n+1}=w_{j}^{n+1 / 2}+G_{\varepsilon, \Delta t}\left(w_{j}^{n+1 / 2}, z_{j}^{n+1 / 2}\right), \\
z_{j}^{n+1}=z_{j}^{n+1 / 2}-G_{\varepsilon, \Delta t}\left(w_{j}^{n+1 / 2}, z_{j}^{n+1 / 2}\right),
\end{array}\right.
$$

with

$$
\begin{aligned}
G_{\varepsilon, \Delta t}(w, z)= & \left(\frac{z-w}{2}-A\left(-\frac{w+z}{2 \sqrt{a}}\right)\right)\left[1-\left(1+\frac{\beta \Delta t}{\varepsilon}\right) \mathrm{e}^{-\beta \Delta t / \varepsilon}\right] \\
& +\frac{\Delta t}{\varepsilon} \mathrm{e}^{-\beta \Delta t / \varepsilon} \mathcal{R}\left(-\frac{w+z}{2 \sqrt{a}}, \frac{z-w}{2}\right) .
\end{aligned}
$$

The main result of this section is to establish the quasi-monotonicity of the operator $G_{\varepsilon, s}$, which will lead to $L^{\infty}$ an $B V$ estimates.

Lemma 3.1 (Comparison principle). Assume the function $\mathcal{R}$ satisfies (1.2), (1.5) and choose $a>0, \beta>0$ such that (2.6) is verified. Then,

(i) the sub-characteristic condition is satisfied for all $(w, z) \in I\left(N_{0}, a_{0}\right)$, that is,

$$
\left|\frac{\partial_{u} \mathcal{R}}{\partial_{v} \mathcal{R}}(u, v)\right|<\sqrt{a}
$$

where $2 \sqrt{a} u:=-(w+z)$ and $2 v:=z-w ;$

(ii) for all $\varepsilon, s>0$, the source term operator $G_{\varepsilon, s}$ is quasi-monotone on the compact set $I\left(N_{0}, a_{0}\right)$, that is,

$$
\left\{\begin{array}{c}
-1 \leq \partial_{w} G_{\varepsilon, s}(w, z) \leq 0, \forall(w, z) \in I\left(N_{0}, a_{0}\right), \\
0 \leq \partial_{z} G_{\varepsilon, s}(w, z) \leq 1, \quad \forall(w, z) \in I\left(N_{0}, a_{0}\right) ;
\end{array}\right.
$$

(iii) consider for $i=1,2,\left(w_{i}^{n+1}, z_{i}^{n+1}\right)$ two solutions to (3.3) corresponding to two initial data $\left(w_{i}^{n+1 / 2}, z_{i}^{n+1 / 2}\right) \in I\left(N_{0}, a_{0}\right)$. Then there exist $w$ and $z \in \mathbb{R}$ such that $(w, z) \in I\left(N_{0}, a_{0}\right)$ and

$$
\left\{\begin{aligned}
w_{1}^{n+1}-w_{2}^{n+1}= & \left(w_{1}^{n+1 / 2}-w_{2}^{n+1 / 2}\right)\left(1+\partial_{w} G_{\varepsilon, s}\left(w, z_{1}^{n+1 / 2}\right)\right) \\
& +\left(z_{1}^{n+1 / 2}-z_{2}^{n+1 / 2}\right) \partial_{z} G_{\varepsilon, s}\left(w_{2}^{n+1 / 2}, z\right), \\
z_{1}^{n+1}-z_{2}^{n+1}= & \left(z_{1}^{n+1 / 2}-z_{2}^{n+1 / 2}\right)\left(1-\partial_{z} G_{\varepsilon, s}\left(w_{2}^{n+1 / 2}, z\right)\right) \\
& -\left(w_{1}^{n+1 / 2}-w_{2}^{n+1 / 2}\right) \partial_{w} G_{\varepsilon, s}\left(w, z_{1}^{n+1 / 2}\right) .
\end{aligned}\right.
$$

Proof. For any $N_{0}>0$ and $a_{0}>0$, we first observe that for $(w, z) \in I\left(N_{0}, a_{0}\right),|u| \leq V\left(N_{0}, a_{0}\right)$. Therefore, using the assumption (1.5) and the definition (2.3), we get that

$$
\left|\frac{\partial_{u} \mathcal{R}}{\partial_{v} \mathcal{R}}(u, v)\right| \leq \frac{g\left(V\left(N_{0}, a_{0}\right)\right)}{\beta_{0}\left(V\left(N_{0}, a_{0}\right)\right)}<\sqrt{a}
$$

which proves the first assertion $(i)$. 
Now we prove (ii) the quasi-monotonicity property of $G_{\varepsilon, s}$. Computing the partial derivatives of $G_{\varepsilon, s}$, it yields for all $s>0$,

$$
\left\{\begin{array}{l}
\partial_{w} G_{\varepsilon, s}=-\frac{1}{2}\left(1-\frac{A^{\prime}(u)}{\sqrt{a}}\right)\left[1-\left(1+\frac{\beta s}{\varepsilon}\right) \mathrm{e}^{-\beta s / \varepsilon}\right]-\frac{s}{2 \varepsilon} \mathrm{e}^{-\beta s / \varepsilon}\left(\frac{\partial_{u} \mathcal{R}}{\sqrt{a}}+\partial_{v} \mathcal{R}\right) \\
\partial_{z} G_{\varepsilon, s}=+\frac{1}{2}\left(1+\frac{A^{\prime}(u)}{\sqrt{a}}\right)\left[1-\left(1+\frac{\beta s}{\varepsilon}\right) \mathrm{e}^{-\beta s / \varepsilon}\right]+\frac{s}{2 \varepsilon} \mathrm{e}^{-\beta s / \varepsilon}\left(\frac{-\partial_{u} \mathcal{R}}{\sqrt{a}}+\partial_{v} \mathcal{R}\right) .
\end{array}\right.
$$

Hence, from the implicit function theorem we obtain

$$
A^{\prime}(u)=-\frac{\partial_{u} \mathcal{R}(u, A(u))}{\partial_{v} \mathcal{R}(u, A(u))}
$$

and the sub-characteristic condition (3.5), we obtain that for all $(u, v) \in I\left(N_{0}, a_{0}\right), \partial_{w} G_{\varepsilon, s}(w, z) \leq 0$ and $\partial_{z} G_{a, s}(w, z) \geq 0$. Moreover, still using condition (3.5), we also get for all $(w, z) \in I\left(N_{0}, a_{0}\right)$

$$
\left\{\begin{array}{l}
\partial_{w} G_{\varepsilon, s}(w, z) \geq-\left[1-\frac{\beta s}{\varepsilon} \mathrm{e}^{-\beta s / \varepsilon}\right]-\partial_{v} \mathcal{R}(u, v) \frac{s}{\varepsilon} \mathrm{e}^{-\beta s / \varepsilon}, \\
\partial_{z} G_{\varepsilon, s}(w, z) \leq\left[1-\frac{\beta s}{\varepsilon} \mathrm{e}^{-\beta s / \varepsilon}\right]+\partial_{v} \mathcal{R}(u, v) \frac{s}{\varepsilon} \mathrm{e}^{-\beta s / \varepsilon}
\end{array}\right.
$$

Now since $|u| \leq V\left(N_{0}, a_{0}\right)$ and from the choice of the parameter $\beta$ in (2.6), it yields that $\left|\partial_{v} \mathcal{R}(u, v)\right| \leq \beta$. Therefore, we conclude that $-1 \leq \partial_{w} G_{\varepsilon, s}(w, z)$ and $\partial_{z} G_{\varepsilon, s}(w, z) \leq 1$, for all $(w, z) \in I\left(N_{0}, a_{0}\right)$.

Finally (iii) follows from a first order Taylor expansion of $G_{\varepsilon, s}$.

This Lemma allows to obtain the following comparison principle.

Corollary 3.2. Consider for $i=1,2$, two initial data $\left(w_{i}^{n+1 / 2}, z_{i}^{n+1 / 2}\right) \in I\left(N_{0}, a_{0}\right)$ satisfying the monotonicity condition $w_{1}^{n+1 / 2} \leq w_{2}^{n+1 / 2}$ and $z_{1}^{n+1 / 2} \leq z_{2}^{n+1 / 2}$. Then, the numerical solution $\left(w_{i}^{n+1}, z_{i}^{n+1}\right)$, given by (3.3) corresponding to the initial data $\left(w_{i}^{n+1 / 2}, z_{i}^{n+1 / 2}\right)$ for $i=1,2$, satisfies

$$
w_{1}^{n+1} \leq w_{2}^{n+1} \quad \text { and } \quad z_{1}^{n+1} \leq z_{2}^{n+1} .
$$

\section{2. $L^{\infty}$ estimates}

We establish a uniform bound on the numerical solution to the scheme (2.12)-(2.14) with respect to the time-space step $h=(\Delta t, \Delta x)$ such that $(2.7)$ is satisfied.

Proposition 3.3. Consider any $a_{0}>0$ and $N_{0}$ given by (2.5). We assume that the function $\mathcal{R}$ satisfies (1.2), (1.5) and choose $a>0, \beta>0$ such that (2.6) is verified. Moreover, the time step $\Delta t$ satisfies the CFL condition (2.7). Then, for all $n \in \mathbb{N},\left\|u^{n}\right\|_{\infty} \leq V\left(N_{0}, a_{0}\right)$ and $\left\|v^{n}\right\|_{\infty} \leq \sqrt{a} V\left(N_{0}, a_{0}\right)$.

Proof. We proceed in two steps and first construct a particular solution $\left(\bar{w}^{n}, \bar{z}^{n}\right) \in \mathbb{R}^{2}$ to the scheme (3.2)-(3.3) which is uniformly bounded, then we apply the comparison principle on the compact set $I\left(N_{0}, a_{0}\right)$ to prove an $L^{\infty}$ bound on $\left(w^{n}, z^{n}\right)$.

To this aim we choose $R_{0}=(1+\sqrt{a}) N_{0}$ and construct a numerical solution $\left(\bar{w}^{n}, \bar{z}^{n}\right)$ to $(3.2)-(3.3)$ corresponding to the initial data $\left(\bar{w}^{0}, \bar{z}^{0}\right)=\left(R_{0}, R_{0}\right)$, which does not depend on the space variable so that the 
transport step (3.2) is invariant. Then we apply the relaxation scheme (3.3), which yields $\bar{w}^{n}=-\bar{v}^{n}-\sqrt{a} \bar{u}^{n}$ and $\bar{z}^{n}=+\bar{v}^{n}-\sqrt{a} \bar{u}^{n}$, where $\left(\bar{u}^{n}, \bar{v}^{n}\right)$ are only given by

$$
\left\{\begin{aligned}
\bar{u}^{n}= & \bar{u}^{0}=-\frac{R_{0}}{\sqrt{a}}=\left(1+\frac{1}{\sqrt{a}}\right) N_{0}, \\
\bar{v}^{n}= & \left(1+\frac{\beta \Delta t}{\varepsilon}\right) \mathrm{e}^{-\beta \Delta t / \varepsilon} \bar{v}^{n-1}+\left(1-\left(1+\frac{\beta \Delta t}{\varepsilon}\right) \mathrm{e}^{-\beta \Delta t / \varepsilon}\right) A\left(\bar{u}^{0}\right) \\
& -\frac{\Delta t}{\varepsilon} \mathrm{e}^{-\beta \Delta t / \varepsilon} \mathcal{R}\left(\bar{u}^{0}, \bar{v}^{n-1}\right) .
\end{aligned}\right.
$$

Then, we proceed by induction to show that for all $n \in\{0, \ldots, N\}$, we have $\left(\bar{w}^{n}, \bar{z}^{n}\right) \in I\left(N_{0}, a_{0}\right)$.

We assume that $\left(\bar{w}^{n-1}, \bar{z}^{n-1}\right) \in I\left(N_{0}, a_{0}\right)$, for some $n \geq 1$. Let us prove that $\left(\bar{w}^{n}, \bar{z}^{n}\right) \in I\left(N_{0}, a_{0}\right)$. On the one hand since $\bar{u}^{n}=\bar{u}^{0}$, it yields $\left\|\bar{u}^{n}\right\|_{\infty} \leq\left\|\bar{u}^{0}\right\|_{\infty} \leq U\left(N_{0}, a_{0}\right)$, where $U\left(N_{0}, a_{0}\right)$ is given by (2.3).

On the other hand, using a first order Taylor expansion of the source term $\mathcal{R}\left(\bar{u}^{0},.\right)$, we get that there exists $\tilde{v}^{n-1} \in \mathbb{R}$ such that

$$
\begin{aligned}
\bar{v}^{n}= & \left(1+\frac{\beta-\partial_{v} \mathcal{R}\left(\bar{u}^{0}, \tilde{v}^{n-1}\right)}{\varepsilon} \Delta t\right) \mathrm{e}^{-\beta \Delta t / \varepsilon} \bar{v}^{n-1} \\
& +\left(1-\left(1+\frac{\beta-\partial_{v} \mathcal{R}\left(\bar{u}^{0}, \tilde{v}^{n-1}\right)}{\varepsilon} \Delta t\right) \mathrm{e}^{-\beta \Delta t / \varepsilon}\right) A\left(\bar{u}^{0}\right) .
\end{aligned}
$$

Therefore, denoting by $\lambda_{k} \in \mathbb{R}$, the real number such that

$$
\lambda_{k}:=\left(1+\frac{\beta-\partial_{v} \mathcal{R}\left(\bar{u}^{0}, \tilde{v}^{k}\right)}{\varepsilon} \Delta t\right) \mathrm{e}^{-\beta \Delta t / \varepsilon}, \quad \forall k \in \mathbb{N},
$$

with $\left|\tilde{v}^{k}\right| \leq F\left(N_{0}, a_{0}\right)$, hence we get $\bar{v}^{n}=\lambda_{n-1} \bar{v}^{n-1}+\left(1-\lambda_{n-1}\right) A\left(\bar{u}^{0}\right)$ and since $\bar{v}^{0}=0$, we have

$$
\bar{v}^{n}=\left(1-\prod_{k=0}^{n-1} \lambda_{k}\right) A\left(\bar{u}^{0}\right) .
$$

Moreover, using that $\left|\bar{u}^{0}\right| \leq U\left(N_{0}, a_{0}\right)$ and $\tilde{v}^{k} \leq \sqrt{a} V\left(N_{0}, a_{0}\right)$, for all $k \in \mathbb{N}$, we get from (1.5) and (2.6),

$$
0<\left(1+\frac{\beta-\beta_{0}}{\varepsilon} \Delta t\right) \mathrm{e}^{-\beta \Delta t / \varepsilon} \leq \lambda_{k} \leq\left(1+\frac{\beta \Delta t}{\varepsilon}\right) \mathrm{e}^{-\beta \Delta t / \varepsilon}<1, \quad \forall k \in \mathbb{N} .
$$

Therefore, $\left\|\bar{v}^{n}\right\|_{\infty} \leq F\left(N_{0}, a_{0}\right)$ and $\left(\bar{w}^{n}, \bar{z}^{n}\right) \in I\left(N_{0}, a_{0}\right)$.

Furthermore, starting from the following initial datum $\left(\underline{w}^{0}, \underline{z}^{0}\right)=\left(-R_{0},-R_{0}\right)$, we can also construct another particular solution $\left(\underline{w}^{n}, \underline{z}^{n}\right) \in I\left(N_{0}, a_{0}\right)$ for all $n \in\{0, \ldots, N\}$.

Now, we proceed to the second step which consists in applying the comparison principle of Corollary 3.2 to prove an $L^{\infty}$ estimate for any initial data $u^{0}, v^{0} \in L^{\infty}(\mathbb{T})$ given by $(2.9)$. From the definition of $N_{0}$, we have $\left\|u^{0}\right\|_{\infty}$ and $\left\|v^{0}\right\|_{\infty} \leq N_{0}$. Then, we have for the initial data $\left\|w^{0}\right\|_{\infty},\left\|z^{0}\right\|_{\infty} \leq(1+\sqrt{a}) N_{0}=R_{0} \leq \sqrt{a} V\left(N_{0}, a_{0}\right)$, that is,

$$
\underline{w}^{0} \leq w^{0} \leq \bar{w}^{0}, \quad \text { and } \quad \underline{z}^{0} \leq z^{0} \leq \bar{z}^{0} .
$$

Thus, we proceed by induction and assume that $\underline{w}^{n} \leq w^{n} \leq \bar{w}^{n}$ and $\underline{z}^{n} \leq z^{n} \leq \bar{z}^{n}$. We first consider the linear transport step (3.2) to $\left(w^{n}, z^{n}\right)$ and get that $\underline{w}^{n} \leq w^{n+1 / 2} \leq \bar{w}^{n}$ and $\underline{z}^{n} \leq z^{n+1 / 2} \leq \bar{z}^{n}$. Thus, we apply Corollary 3.2 to the two solutions to (3.3) associated to the initial conditions $\left(w_{1}^{n+1 / 2}, z_{1}^{n+1 / 2}\right)=$ $\left(w^{n+1 / 2}, z^{n+1 / 2}\right)$ and $\left(w_{2}^{n+1 / 2}, z_{2}^{n+1 / 2}\right)=\left(\underline{w}^{n}, \underline{z}^{n}\right)$ (and then $\left.\left(\bar{w}^{n}, \bar{z}^{n}\right)\right)$, we have

$$
\underline{w}^{n+1} \leq w^{n+1} \leq \bar{w}^{n+1} \quad \text { and } \quad \underline{\mathrm{z}}^{\mathrm{n}+1} \leq \mathrm{z}^{\mathrm{n}+1} \leq \overline{\mathrm{z}}^{\mathrm{n}+1},
$$


which finally gives for all $n \in \mathbb{N}$, that $\left(w^{n}, z^{n}\right) \in I\left(N_{0}, a_{0}\right)$. By construction of $\left(u^{n}, v^{n}\right)$ we have proved that $\left\|u^{n}\right\|_{\infty} \leq V\left(N_{0}, a_{0}\right)$ and $\left\|v^{n}\right\|_{\infty} \leq \sqrt{a} V\left(N_{0}, a_{0}\right)$.

\section{3. $B V$ estimates}

In this section, we obtain a $B V$ estimate on the numerical solution to the scheme (2.12)-(2.14) with the time-space step $h=(\Delta t, \Delta x)$ such that $(2.7)$ is satisfied.

Proposition 3.4. Assume that $u_{0}, v_{0}$ are uniformly bounded with respect to $\varepsilon$ in $B V(\mathbb{T})$. For any $a_{0}>0$ and $N_{0}$ given by (2.5), we assume that the function $\mathcal{R}$ satisfies (1.2), (1.5) and choose a $>0, \beta>0$ such that (2.6) is verified and $h$ satisfies (2.7). Then, for all $n \in \mathbb{N}$, we have:

$$
T V\left(w^{n+1}\right)+T V\left(z^{n+1}\right) \leq T V\left(w^{n}\right)+T V\left(z^{n}\right) .
$$

The proof is straightforward applying (iii) of Lemma 3.1 to initial conditions $\left(w_{j}^{n+1 / 2}, z_{j}^{n+1 / 2}\right)$ and $\left(w_{j+1}^{n+1 / 2}, z_{j+1}^{n+1 / 2}\right)$.

\section{Trend to Equilibrium (Proof of Theorem 2.2)}

For a sequence $u=\left(u_{j}\right)_{j \in \mathbb{Z}}$ we set

$$
\|u\|_{1}:=\sum_{j \in \mathbb{Z}} \Delta x\left|u_{j}\right|
$$

In this section we first focus on the asymptotic behavior of the numerical solution to (2.12)-(2.14) when $\varepsilon$ goes to zero or when times goes to infinity. Then, we prove that this numerical solution converges to a consistent approximation of the conservation laws (1.3) when $\varepsilon$ goes to zero.

\subsection{Asymptotic behavior}

In this subsection, we drop the subscripts $\varepsilon$ for sake of clarity and estimate the deviation to the local equilibrium $\delta^{n}=v^{n}-A\left(u^{n}\right)$.

Proposition 4.1. Assume that $u_{0}, v_{0}$ are uniformly bounded with respect to $\varepsilon$ in $B V(\mathbb{T})$. For any $a_{0}>0$ and $N_{0}$ given by (2.5), we assume that the function $\mathcal{R}$ satisfies (1.2), (1.5) and choose $a>0, \beta>0$ such that (2.6) is verified and $h$ satisfies (2.7). Then the deviation from the equilibrium, $\delta=v-A(u)$ satisfies for all $n \in \mathbb{N}$ and all $\varepsilon>0$

$$
\left\{\begin{array}{l}
\left\|\delta^{n+1 / 2}\right\|_{1} \leq\left\|\delta^{n}\right\|_{1}+C \Delta t, \\
\left\|\delta^{n}\right\|_{1} \leq \mathrm{e}^{-\beta_{0} t^{n} / \varepsilon}\left\|\delta^{0}\right\|_{1}+C \varepsilon .
\end{array}\right.
$$

where $C>0$ is a constant only depending on the parameters $a, \beta_{0}$ and the $B V$ norm of the initial data.

Moreover, if $\varepsilon<\Delta t$ then we get

$$
\left\|\delta^{n}\right\|_{1} \leq \mathrm{e}^{-\beta_{0} t^{n} / \varepsilon}\left\|\delta^{0}\right\|_{1}+C_{a} \Delta t \mathrm{e}^{-\beta_{0} \Delta t / \varepsilon} .
$$

Proof. We set for $j \in \mathbb{Z}, n \in \mathbb{N}$ the sequence of deviations from the equilibrium:

$$
\delta_{j}^{n}=v_{j}^{n}-A\left(u_{j}^{n}\right) .
$$

We first consider the transport step (2.12) of the numerical scheme: for all $j \in \mathbb{Z}$, we apply a Taylor expansion to $A$, then there exists $\xi_{j}^{n}$ such that $\left|\xi_{j}^{n}\right| \leq V\left(N_{0}, a_{0}\right)$ and

$$
\begin{aligned}
\delta_{j}^{n+1 / 2}= & \delta_{j}^{n}-\frac{\Delta t}{2 \Delta x}\left[a\left(u_{j+1}^{n}-u_{j-1}^{n}\right)-\sqrt{a}\left(v_{j+1}^{n}-2 v_{j}^{n}+v_{j-1}^{n}\right)\right] \\
& -\frac{\Delta t}{2 \Delta x} A^{\prime}\left(\xi_{j}^{n}\right)\left[\left(v_{j+1}^{n}-v_{j-1}^{n}\right)-\sqrt{a}\left(u_{j+1}^{n}-2 u_{j}^{n}+u_{j-1}^{n}\right)\right] .
\end{aligned}
$$


Thanks to the uniform $B V$ estimate, proved in Proposition 3.4, the sub-characteristic condition $\left|A^{\prime}\left(\xi_{j}^{n}\right)\right|<\sqrt{a}$ and the TVD property of the numerical fluxes we get the first estimate (4.1), by multiplying by $\Delta x$ and summing over $j \in \mathbb{Z}$ :

$$
\left\|\delta^{n+1 / 2}\right\|_{1} \leq\left\|\delta^{n}\right\|_{1}+\Delta t C_{a}\left[T V\left(v^{0}\right)+\sqrt{a} T V\left(u^{0}\right)\right],
$$

where $C_{a}>0$ is a constant only depending on $a$.

Then, we consider the second step of the numerical scheme (2.14). On the one hand, since $u^{n+1}=u^{n+1 / 2}$, it yields

$$
\delta_{j}^{n+1}=\delta_{j}^{n+1 / 2}\left[1+\beta \frac{\Delta t}{\varepsilon}\right] \mathrm{e}^{-\beta \Delta t / \varepsilon}-\frac{\Delta t}{\varepsilon} \mathrm{e}^{-\beta \Delta t / \varepsilon} \mathcal{R}\left(u_{j}^{n+1 / 2}, v_{j}^{n+1 / 2}\right) .
$$

On the other hand, applying a Taylor expansion, since $\mathcal{R}(u, A(u))=0$ we get that there exists $\eta$ such that $|\eta| \leq \sqrt{a} V\left(N_{0}, a_{0}\right)$ and:

$$
\mathcal{R}\left(u_{j}^{n+1 / 2}, v_{j}^{n+1 / 2}\right)=\partial_{v} \mathcal{R}\left(u_{j}^{n+1 / 2}, \eta\right) \delta_{j}^{n+1 / 2} .
$$

Hence, we have

$$
\delta_{j}^{n+1}=\delta_{j}^{n+1 / 2}\left[1+\left(1-\frac{\partial_{v} \mathcal{R}\left(u_{j}^{n+1 / 2}, \eta\right)}{\beta}\right) \frac{\beta \Delta t}{\varepsilon}\right] \mathrm{e}^{-\beta \Delta t / \varepsilon} .
$$

Therefore under the assumption (1.5), we set for all $s \geq 0$

$$
g(s)=\left[1+\left(1-\frac{\beta_{0}}{\beta}\right) s\right] \mathrm{e}^{-s},
$$

for which we easily show that for all $s \in \mathbb{R}^{+}$, we have that $\mathrm{e}^{-s} \leq g(s) \leq \mathrm{e}^{-\beta_{0} s / \beta}$. Hence, taking $s=\beta \Delta t / \varepsilon$

$$
\left\|\delta^{n+1}\right\|_{1} \leq \mathrm{e}^{-\beta_{0} \Delta t / \varepsilon}\left\|\delta^{n+1 / 2}\right\|_{1}
$$

Finally, gathering (4.3) and (4.4), we obtain that there exists a constant $C_{1}>0$ depending only on $a, T V\left(u^{0}\right)$ and $T V\left(v^{0}\right)$ such that $\left\|\delta^{n+1}\right\|_{1} \leq \mathrm{e}^{-\beta_{0} \Delta t / \varepsilon}\left[\left\|\delta^{n}\right\|_{1}+C_{1} \Delta t\right]$. By induction, we easily get

$$
\left\|\delta^{n}\right\|_{1} \leq \mathrm{e}^{-\beta_{0} t^{n} / \varepsilon}\left\|\delta^{0}\right\|_{1}+C_{a} \Delta t \frac{\mathrm{e}^{-\beta_{0} \Delta t / \varepsilon}}{1-\mathrm{e}^{-\beta_{0} \Delta t / \varepsilon}}
$$

To conclude we only observe that $x \mathrm{e}^{-x} \leq 1-\mathrm{e}^{-x}$, for any $x \geq 0$, then it gives the second estimate of (4.1): there exists a constant $C>0$, only depending on $a, \beta_{0}, T V\left(u^{0}\right)$ and $T V\left(v^{0}\right)$ such that $\left\|\delta^{n}\right\|_{1} \leq \mathrm{e}^{-\beta_{0} t^{n} / \varepsilon}\left\|\delta^{0}\right\|_{1}+C \varepsilon$. Moreover, when $\varepsilon<\Delta t$, we again start from the estimate (4.5) and note that $1 /\left(1-\mathrm{e}^{-\beta_{0} \Delta t / \varepsilon}\right) \leq 1 /\left(1-\mathrm{e}^{-\beta_{0}}\right)$. Thus, there exists another constant $C>0$, only depending on $a, \beta_{0}, T V\left(u^{0}\right)$ and $T V\left(v^{0}\right)$ such that

$$
\left\|\delta^{n}\right\|_{1} \leq \mathrm{e}^{-\beta_{0} t^{n} / \varepsilon}\left\|\delta^{0}\right\|_{1}+C \Delta t \mathrm{e}^{-\beta_{0} \Delta t / \varepsilon}
$$

which gives (4.2).

\subsection{Proof of Theorem 2.2}

We are now ready to perform the asymptotic analysis of the numerical scheme $(2.12)-(2.14)$ when $\varepsilon$ goes to zero. We keep now the subscript $\varepsilon$ for the solution to the relaxation system.

Let us consider the numerical solution $\left(u_{h}^{\varepsilon}, v_{h}^{\varepsilon}\right)$ to the scheme $(2.12)-(2.14)$ written in the form (3.2)-(3.3) with

$$
w_{h}^{\varepsilon}=-v_{h}^{\varepsilon}-\sqrt{a} u_{h}^{\varepsilon} \quad \text { and } \quad z_{h}^{\varepsilon}=+v_{h}^{\varepsilon}-\sqrt{a} u_{h}^{\varepsilon},
$$


such that

$$
\left\{\begin{array}{l}
w_{h}^{\varepsilon}(t, x)=\sum_{n \in \mathbb{N}} \sum_{j \in \mathbb{Z}} w_{j}^{\varepsilon, n} \mathbf{1}_{C_{j}}(x) \mathbf{1}_{\left[t^{n}, t^{n+1}[\right.}(t), \\
z_{h}^{\varepsilon}(t, x)=\sum_{n \in \mathbb{N}} \sum_{j \in \mathbb{Z}} z_{j}^{\varepsilon, n} \mathbf{1}_{C_{j}}(x) \mathbf{1}_{\left[t^{n}, t^{n+1}[\right.}(t),
\end{array}\right.
$$

where $\left(w_{j}^{n}, z_{j}^{n}\right)_{j, n}$ is given by $(3.2)-(3.3)$. Let us also define $\left(w_{h}, z_{h}\right)$ the numerical solution to the limit of the scheme (3.2)-(3.3), when $\varepsilon \rightarrow 0$. Namely, $w_{h}$ and $z_{h}$ are given by

$$
\left\{\begin{array}{l}
w_{h}(t, x)=\sum_{n \in \mathbb{N}} \sum_{j \in \mathbb{Z}} w_{j}^{n} \mathbf{1}_{C_{j}}(x) \mathbf{1}_{\left[t^{n}, t^{n+1}[\right.}(t), \\
z_{h}(t, x)=\sum_{n \in \mathbb{N}} \sum_{j \in \mathbb{Z}} z_{j}^{n} \mathbf{1}_{C_{j}}(x) \mathbf{1}_{\left[t^{n}, t^{n+1}[\right.}(t)
\end{array}\right.
$$

where $\left(w_{j}^{n}, z_{j}^{n}\right)$ is given by the formal cancellation of $\varepsilon$ in (3.2)-(3.3). To obtain an error estimates we rewrite the values $\left(w_{j}^{n}, z_{j}^{n}\right)_{(n, j) \in \mathbb{N} \times \mathbb{Z}}$ as a perturbation of the numerical solution to (3.2)-(3.3) with a fixed value of $\varepsilon>0$ :

$$
\left\{\begin{array}{l}
w_{j}^{n+1 / 2}=w_{j}^{n}-\sqrt{a} \frac{\Delta t}{\Delta x}\left(w_{j}^{n}-w_{j-1}^{n}\right), \\
z_{j}^{n+1 / 2}=z_{j}^{n}+\sqrt{a} \frac{\Delta t}{\Delta x}\left(z_{j+1}^{n}-z_{j}^{n}\right),
\end{array}\right.
$$

and then

$$
\left\{\begin{array}{l}
w_{j}^{n+1}=w_{j}^{n+1 / 2}+G_{\varepsilon, \Delta t}\left(w_{j}^{n+1 / 2}, z_{j}^{n+1 / 2}\right)-\Delta t \mathcal{E}_{j}^{n}(\varepsilon), \\
z_{j}^{n+1}=z_{j}^{n+1 / 2}-G_{\varepsilon, \Delta t}\left(w_{j}^{n+1 / 2}, z_{j}^{n+1 / 2}\right)+\Delta t \mathcal{E}_{j}^{n}(\varepsilon) .
\end{array}\right.
$$

where $\Delta t \mathcal{E}_{j}^{n}(\varepsilon)$ represents the consistency error of the operator $G_{\varepsilon, \Delta t}$ with respect to $\varepsilon$, that is,

$$
\Delta t \mathcal{E}_{j}^{n}(\varepsilon):=G_{\varepsilon, \Delta t}\left(w_{j}^{n+1 / 2}, z_{j}^{n+1 / 2}\right)-G_{0, \Delta t}\left(w_{j}^{n+1 / 2}, z_{j}^{n+1 / 2}\right) .
$$

Therefore, we apply Lemma 3.1 (ii) and (iii) in (4.6), with $\left(w_{1}, z_{1}\right)=\left(w_{j}^{\varepsilon}, z_{j}^{\varepsilon}\right)$ and $\left(w_{2}, z_{2}\right)=\left(w_{j}, z_{j}\right)$, it yields

$$
\left|w_{j}^{\varepsilon, n+1}-w_{j}^{n+1}\right|+\left|z_{j}^{\varepsilon, n+1}-z_{j}^{n+1}\right| \leq\left|w_{j}^{\varepsilon, n+1 / 2}-w_{j}^{n+1 / 2}\right|+\left|z_{j}^{\varepsilon, n+1 / 2}-z_{j}^{n+1 / 2}\right|+2\left|\Delta t \mathcal{E}_{j}^{n}(\varepsilon)\right|,
$$

and by linearity of the transport scheme (3.2), we have for all $n \geq 0$

$$
\left|w_{j}^{\varepsilon, n+1}-w_{j}^{n+1}\right|+\left|z_{j}^{\varepsilon, n+1}-z_{j}^{n+1}\right| \leq\left|w_{j}^{\varepsilon, n}-w_{j}^{n}\right|+\left|z_{j}^{\varepsilon, n}-z_{j}^{n}\right|+2\left|\Delta t \mathcal{E}_{j}^{n}(\varepsilon)\right| .
$$

Thus, multiplying by $\Delta x$, summing over $j \in \mathbb{Z}$ and applying a straightforward induction, we get the following stability result

$$
\sum_{j \in \mathbb{Z}} \Delta x\left(\left|w_{j}^{\varepsilon, n}-w_{j}^{n}\right|+\left|z_{j}^{\varepsilon, n}-z_{j}^{n}\right|\right) \leq \sum_{j \in \mathbb{Z}} \Delta x\left(\left|w_{j}^{\varepsilon, 0}-w_{j}^{0}\right|+\left|z_{j}^{\varepsilon, 0}-z_{j}^{0}\right|\right)+2 \sum_{k=0}^{n-1} \sum_{j \in \mathbb{Z}} \Delta t \Delta x\left|\mathcal{E}_{j}^{k}(\varepsilon)\right| .
$$

It now remains to evaluate the error $\mathcal{E}_{j}^{n}(\varepsilon)$. Using that for any $(w, z) \in I\left(N_{0}, a_{0}\right)$, the function $\mathcal{R} \in \mathcal{C}^{1}\left(\mathbb{R}^{+}, \mathbb{R}\right)$ verifies $\beta_{0} \leq \partial_{v} \mathcal{R}(u, v) \leq \beta$, we have

$$
\begin{aligned}
\Delta t\left|\mathcal{E}_{j}^{n}(\varepsilon)\right| & =\mathrm{e}^{-\beta \Delta t / \varepsilon}\left|-\left(v_{j}^{n+1 / 2}-A\left(u_{j}^{n+1 / 2}\right)\right)\left(1+\frac{\beta \Delta t}{\varepsilon}\right)+\frac{\Delta t}{\varepsilon} \mathcal{R}\left(u_{j}^{n+1 / 2}, v_{j}^{n+1 / 2}\right)\right|, \\
& \leq \mathrm{e}^{-\beta_{0} \Delta t / \varepsilon}\left|v_{j}^{n+1 / 2}-A\left(u_{j}^{n+1 / 2}\right)\right| .
\end{aligned}
$$


Thanks to the estimates (4.1) and (4.2) in Proposition 4.1 on the deviation applied to $v^{n+1 / 2}-A\left(u^{n+1 / 2}\right)$ which is also valid in the asymptotic $\varepsilon \rightarrow 0$, it yields

$$
\left\|v^{n+1 / 2}-A\left(u^{n+1 / 2}\right)\right\|_{1} \leq \begin{cases}\left\|\delta^{0}\right\|_{1}+C \Delta t & \text { if } n=0 \\ C \Delta t & \text { if } n>0 .\end{cases}
$$

Then, we get for $k \geq 0$ and $\varepsilon \leq \Delta t$,

$$
\sum_{j \in \mathbb{Z}} \Delta x \Delta t\left|\mathcal{E}_{j}^{k}(\varepsilon)\right| \leq \mathrm{e}^{-\beta_{0} \Delta t / \varepsilon}\left[\left\|\delta^{0}\right\|_{1}+C \Delta t\right]
$$

Hence summing over $0 \leq k \leq n$, it gives

$$
\sum_{k=0}^{n} \sum_{j \in \mathbb{Z}} \Delta x \Delta t\left|\mathcal{E}_{\varepsilon, \Delta t}^{k}\right| \leq \mathrm{e}^{-\beta_{0} \Delta t / \varepsilon}\left[\left\|\delta^{0}\right\|_{1}+C t^{n}\right] .
$$

Finally, we get the estimate

$$
\begin{aligned}
\left\|w_{h}^{\varepsilon}\left(t^{n}\right)-w_{h}\left(t^{n}\right)\right\|_{1}+\left\|z_{h}^{\varepsilon}\left(t^{n}\right)-z_{h}\left(t^{n}\right)\right\|_{1} \leq & \left\|w_{h}^{\varepsilon}(0)-w_{h}(0)\right\|_{1}+\left\|z_{h}^{\varepsilon}(0)-z_{h}(0)\right\|_{1} \\
& +2 \mathrm{e}^{-\beta_{0} \Delta t / \varepsilon}\left[\left\|\delta^{0}\right\|_{1}+C t^{n-1}\right]
\end{aligned}
$$

and the result follows $\left(u_{h}^{\varepsilon}, v_{h}^{\varepsilon}\right) \rightarrow\left(u_{h}, v_{h}\right)$, when $\varepsilon$ goes to zero. The Proof of Theorem 2.2 is now complete.

\section{Proof of Theorem 2.3}

In this section, we prove the convergence of the relaxation Asymptotic Preserving scheme. As in the stability analysis of the relaxation scheme, we will rather consider the diagonal variables $w$ and $z$ and drop the subscripts $\varepsilon$ for sake of clarity when it is not necessary.

\subsection{Consistency error}

Consider $(u, v)$ the exact solution to (1.1) and define $(w, z)$ by (3.1). Unfortunately, this solution is not smooth enough to study the consistency error, then we introduce a regularization $\left(w_{\delta}, z_{\delta}\right)$ given by

$$
\left\{\begin{array}{l}
w_{\delta}(t, x)=w \star \rho_{\delta}(t, x) \\
z_{\delta}(t, x)=z \star \rho_{\delta}(t, x)
\end{array}\right.
$$

where $\star$ denotes the convolution product with respect to $x \in \mathbb{T}$ and

$$
\rho_{\delta}(x)=\frac{1}{\delta} \rho\left(\frac{x}{\delta}\right) \quad \text { and } \quad \rho \in \mathcal{C}_{c}^{\infty}(\mathbb{T}), \quad \rho \geq 0, \quad \int_{\mathbb{T}} \rho(z) \mathrm{d} z=1 .
$$

Thus, the couple $\left(w_{\delta}, z_{\delta}\right)$ is solution to

$$
\left\{\begin{array}{l}
\partial_{t} w_{\delta}+\sqrt{a} \partial_{x} w_{\delta}=+\frac{1}{\varepsilon} \mathcal{R}_{\delta}(u, v), \\
\partial_{t} z_{\delta}-\sqrt{a} \partial_{x} z_{\delta}=-\frac{1}{\varepsilon} \mathcal{R}_{\delta}(u, v)
\end{array}\right.
$$


with $\mathcal{R}_{\delta}=\mathcal{R} \star \rho_{\delta}$ and $(u, v)$ solution to (1.1). Therefore, applying the Duhamel's representation, the solution can be written as

$$
\left\{\begin{array}{l}
w_{\delta}\left(t^{n+1}, x\right)=w_{\delta}\left(t^{n}, x-\sqrt{a} \Delta t\right)+\frac{1}{\varepsilon} \int_{0}^{\Delta t} \mathcal{R}_{\delta}(u, v)\left(t^{n}+s, x-\sqrt{a}(\Delta t-s)\right) \mathrm{d} t \\
z_{\delta}\left(t^{n+1}, x\right)=z_{\delta}\left(t^{n}, x+\sqrt{a} \Delta t\right)-\frac{1}{\varepsilon} \int_{0}^{\Delta t} \mathcal{R}_{\delta}(u, v)\left(t^{n}+s, x+\sqrt{a}(\Delta t-s)\right) \mathrm{d} t
\end{array}\right.
$$

Then we set

$$
\tilde{w}_{j}^{n}=\frac{1}{\Delta x} \int_{C_{j}} w_{\delta}\left(t^{n}, x\right) \mathrm{d} x, \quad \tilde{z}_{j}^{n}=\frac{1}{\Delta x} \int_{C_{j}} z_{\delta}\left(t^{n}, x\right) \mathrm{d} x .
$$

Integrating (5.2) over $x \in C_{j}$ and dividing by $\Delta x$, it yields

$$
\left\{\begin{array}{l}
\tilde{w}_{j}^{n+1}=\tilde{w}_{j}^{n+1 / 2}+G_{\varepsilon, \Delta t}\left(\tilde{w}_{j}^{n+1 / 2}, \tilde{z}_{j}^{n+1 / 2}\right)+\Delta t \mathcal{E}_{1, j}^{n}+\Delta t \mathcal{E}_{2, j}^{n}, \\
\tilde{z}_{j}^{n+1}=\tilde{z}_{j}^{n+1 / 2}-G_{\varepsilon, \Delta t}\left(\tilde{w}_{j}^{n+1 / 2}, \tilde{z}_{j}^{n+1 / 2}\right)+\Delta t \mathcal{E}_{3, j}^{n}+\Delta t \mathcal{E}_{4, j}^{n}
\end{array}\right.
$$

with

$$
\left\{\begin{array}{l}
\tilde{w}_{j}^{n+1 / 2}=\tilde{w}_{j}^{n}-\sqrt{a} \frac{\Delta t}{\Delta x}\left(\tilde{w}_{j}^{n}-\tilde{w}_{j-1}^{n}\right) \\
\tilde{z}_{j}^{n+1 / 2}=\tilde{z}_{j}^{n}+\sqrt{a} \frac{\Delta t}{\Delta x}\left(\tilde{z}_{j+1}^{n}-\tilde{z}_{j}^{n}\right) .
\end{array}\right.
$$

The consistency errors related to the transport operator $\mathcal{E}_{1, j}^{n}, \mathcal{E}_{3, j}^{n}$ are respectively defined by

$$
\Delta t \mathcal{E}_{1, j}^{n}=\frac{\varepsilon_{1, j+1 / 2}^{n}-\varepsilon_{1, j-1 / 2}^{n}}{\Delta x}, \quad \Delta t \mathcal{E}_{3, j}^{n}=\frac{\varepsilon_{3, j+1 / 2}^{n}-\varepsilon_{3, j-1 / 2}^{n}}{\Delta x},
$$

where $\varepsilon_{1, j+1 / 2}^{n}$ and $\varepsilon_{3, j+1 / 2}^{n}$ are the consistency errors of the numerical flux and are given by

$$
\left\{\begin{array}{l}
\varepsilon_{1, j+1 / 2}^{n}=-\int_{0}^{\sqrt{a} \Delta t} w_{\delta}\left(t^{n}, x_{j+1 / 2}-s\right) \mathrm{d} s+\sqrt{a} \Delta t \tilde{w}_{j}^{n}, \\
\varepsilon_{3, j+1 / 2}^{n}=+\int_{0}^{\sqrt{a} \Delta t} z_{\delta}\left(t^{n}, x_{j+1 / 2}+s\right) \mathrm{d} s-\sqrt{a} \Delta t \tilde{z}_{j+1}^{n},
\end{array}\right.
$$

whereas the consistency errors $\Delta t \mathcal{E}_{2, j}^{n}$ and $\Delta t \mathcal{E}_{4, j}^{n}$ correspond to the stiff source term and are given by

$$
\left\{\begin{array}{l}
\Delta t \mathcal{E}_{2, j}^{n}=+\frac{1}{\Delta x} \int_{C_{j}} \int_{0}^{\Delta t} \frac{1}{\varepsilon} \mathcal{R}_{\delta}(u, v)\left(t^{n}+s, x-\sqrt{a}(\Delta t-s)\right) \mathrm{d} s-G_{\varepsilon, \Delta t}\left(\tilde{w}_{j}^{n+1 / 2}, \tilde{z}_{j}^{n+1 / 2}\right) \mathrm{d} x, \\
\Delta t \mathcal{E}_{4, j}^{n}=-\frac{1}{\Delta x} \int_{C_{j}} \int_{0}^{\Delta t} \frac{1}{\varepsilon} \mathcal{R}_{\delta}(u, v)\left(t^{n}+s, x+\sqrt{a}(\Delta t-s)\right) \mathrm{d} s-G_{\varepsilon, \Delta t}\left(\tilde{w}_{j}^{n+1 / 2}, \tilde{z}_{j}^{n+1 / 2}\right) \mathrm{d} x .
\end{array}\right.
$$

We then evaluate successively each consistency error term. On the one hand, we prove the following consistency error for smooth solutions, which is related to the transport approximation.

Proposition 5.1. Let $(w, z)$ be given by (3.1), where $(u, v)$ is the exact solution to (1.1) and such that $w$, $z \in L^{\infty}\left(\mathbb{R}^{+}, B V(\mathbb{T})\right)$. Then the consistency error related to the transport part satisfies

$$
\sum_{j \in \mathbb{Z}} \Delta x\left[\left|\mathcal{E}_{1, j}^{n}\right|+\left|\mathcal{E}_{3, j}^{n}\right|\right] \leq C \frac{\Delta x}{\delta}\left(T V\left(w\left(t^{n}\right)\right)+T V\left(z\left(t^{n}\right)\right) .\right.
$$


Proof. We first study the consistency error for $w \in L^{\infty}\left(\mathbb{R}^{+}, B V(\mathbb{T})\right)$. We perform a simple change of variable in (5.5), which yields since $\sqrt{a} \Delta t=\lambda \Delta x$,

$$
\begin{aligned}
\varepsilon_{1, j+1 / 2}^{n} & =-\lambda \int_{0}^{\Delta x} w_{\delta}\left(t^{n}, x_{j+1 / 2}-\lambda s\right) \mathrm{d} s+\lambda \int_{0}^{\Delta x} w_{\delta}\left(t^{n}, x_{j+1 / 2}-s\right) \mathrm{d} s, \\
& =\lambda \int_{0}^{\Delta x} \int_{\lambda s}^{s} \partial_{x} w_{\delta}\left(t^{n}, x_{j+1 / 2}-r\right) \mathrm{d} r \mathrm{~d} s .
\end{aligned}
$$

Therefore, since $w_{\delta}$ is smooth we have

$$
\begin{aligned}
\left|\mathcal{E}_{1, j}^{n}\right| & =\frac{\sqrt{a}}{\Delta x^{2}}\left|\int_{0}^{\Delta x} \int_{\lambda s}^{s} \partial_{x} w_{\delta}\left(t^{n}, x_{j+1 / 2}-r\right)-\partial_{x} w_{\delta}\left(t^{n}, x_{j-1 / 2}-r\right) \mathrm{d} r \mathrm{~d} s\right|, \\
& \leq \sqrt{a} \int_{x_{i-3 / 2}}^{x_{i+1 / 2}}\left|\partial_{x x}^{2} w_{\delta}\left(t^{n}, x\right)\right| \mathrm{d} x .
\end{aligned}
$$

By multiplying by $\Delta x$ and summing over $j \in \mathbb{Z}$, we get an estimate for a smooth solution $w_{\delta}\left(t^{n}\right) \in W^{2,1}(\mathbb{T})$,

$$
\sum_{j \in \mathbb{Z}} \Delta x\left|\mathcal{E}_{1, j}^{n}\right| \leq 2 \sqrt{a} \Delta x\left\|\partial_{x x}^{2} w_{\delta}\left(t^{n}\right)\right\|_{1} .
$$

To achieve the proof, we need to estimate $\left\|\partial_{x x}^{2} w_{\delta}\left(t^{n}\right)\right\|_{1}$ with respect to $w$ and $\rho_{\delta}$. Using the convolution properties, we easily get

$$
\left\|\partial_{x x}^{2} w_{\delta}\left(t^{n}\right)\right\|_{1} \leq \frac{C}{\delta}\left\|\partial_{x} w_{\delta}\left(t^{n}\right)\right\|_{1} \leq \frac{C}{\delta} T V\left(w\left(t^{n}\right)\right),
$$

which allows to conclude that

$$
\sum_{j \in \mathbb{Z}} \Delta x\left|\mathcal{E}_{1, j}^{n}\right| \leq C \frac{\Delta x}{\delta} T V\left(w\left(t^{n}\right)\right)
$$

Using a similar technique, we also get for a smooth solution $z \in L^{\infty}\left(\mathbb{R}^{+}, B V(\mathbb{T})\right)$,

$$
\sum_{j \in \mathbb{Z}} \Delta x\left|\mathcal{E}_{3, j}^{n}\right| \leq C \frac{\Delta x}{\delta} T V\left(z\left(t^{n}\right)\right)
$$

On the other hand, we treat the consistency errors $\mathcal{E}_{2, j}^{n}$ and $\mathcal{E}_{4, j}^{n}$, which are related to the stiff source term.

Proposition 5.2. Let $(w, z)$ be given by (3.1), where $(u, v)$ is the exact solution to (1.1). Assume that $w$, $z \in L^{\infty}\left(\mathbb{R}^{+}, B V(\mathbb{T})\right)$. Then there exists a constant $C>0$, only depending on $u$ and $v$ such that the consistency error related to the stiff source part satisfies

$$
\sum_{j \in \mathbb{Z}} \Delta x\left|\mathcal{E}_{2, j}^{n}\right| \leq C\left[\frac{\Delta t}{\varepsilon}\left(\mathrm{e}^{-\beta_{0} t^{n} / \varepsilon} \frac{\left\|\delta^{0}\right\|_{1}}{\varepsilon}+1\right)+\frac{\Delta x}{\varepsilon}+\frac{\delta}{\varepsilon}\right]
$$

and

$$
\sum_{j \in \mathbb{Z}} \Delta x\left|\mathcal{E}_{4, j}^{n}\right| \leq C\left[\frac{\Delta t}{\varepsilon}\left(\mathrm{e}^{-\beta_{0} t^{n} / \varepsilon} \frac{\left\|\delta^{0}\right\|_{1}}{\varepsilon}+1\right)+\frac{\Delta x}{\varepsilon}+\frac{\delta}{\varepsilon}\right] .
$$

Proof. We first define $\left(\tilde{u}_{j}^{n}, \tilde{v}_{j}^{n}\right)$ such that $2 \sqrt{a} \tilde{u}_{j}^{n}=-\left(\tilde{w}_{j}^{n}+\tilde{z}_{j}^{n}\right)$ and $2 \tilde{v}_{j}^{n}=\tilde{z}_{j}^{n}-\tilde{w}_{j}^{n}$ Therefore, we split the consistency error $\mathcal{E}_{2, j}^{n}$ as

$$
\mathcal{E}_{2, j}^{n}=\mathcal{E}_{21, j}^{n}+\mathcal{E}_{22, j}^{n}+\mathcal{E}_{23, j}^{n}+\mathcal{E}_{24, j}^{n}+\mathcal{E}_{25, j}^{n}
$$


with

$$
\left\{\begin{array}{l}
\Delta t \mathcal{E}_{21, j}^{n}=-\left[1-\left(1+\frac{\beta \Delta t}{\varepsilon}\right) \mathrm{e}^{-\beta \Delta t / \varepsilon}\right]\left(\tilde{v}_{j}^{n+1 / 2}-A\left(\tilde{u}_{j}^{n+1 / 2}\right)\right), \\
\Delta t \mathcal{E}_{22, j}^{n}=\left(1-\mathrm{e}^{-\beta \Delta t / \varepsilon}\right) \frac{\Delta t}{\varepsilon} \mathcal{R}\left(\tilde{u}_{j}^{n+1 / 2}, \tilde{v}_{j}^{n+1 / 2}\right), \\
\Delta t \mathcal{E}_{23, j}^{n}=\frac{1}{\varepsilon \Delta x} \int_{C_{j}} \int_{0}^{\Delta t} \mathcal{R}_{\delta}(u, v)\left(t^{n}+s, x-\sqrt{a}(\Delta t-s)\right)-\mathcal{R}_{\delta}(u, v)\left(t^{n}, x-\sqrt{a}(\Delta t)\right) \mathrm{d} s \mathrm{~d} x, \\
\Delta t \mathcal{E}_{24, j}^{n}=\frac{\Delta t}{\varepsilon \Delta x} \int_{C_{j}} \mathcal{R}_{\delta}(u, v)\left(t^{n}, x-\sqrt{a}(\Delta t)\right)-\mathcal{R}(u, v)\left(t^{n}, x-\sqrt{a}(\Delta t)\right) \mathrm{d} x, \\
\Delta t \mathcal{E}_{25, j}^{n}=\frac{\Delta t}{\varepsilon \Delta x} \int_{C_{j}} \mathcal{R}(u, v)\left(t^{n}, x-\sqrt{a}(\Delta t)\right)-\mathcal{R}\left(\tilde{u}_{j}^{n+1 / 2}, \tilde{v}_{j}^{n+1 / 2}\right) \mathrm{d} x .
\end{array}\right.
$$

On the one hand, the two terms $\mathcal{E}_{21, j}^{n}$ and $\mathcal{E}_{22, j}^{n}$ can be easily evaluated using a Taylor expansion of $s \mapsto \mathrm{e}^{-\beta s / \varepsilon}$, it yields

$$
\Delta t\left|\mathcal{E}_{21, j}^{n}\right| \leq \frac{1}{2}\left(\frac{\beta \Delta t}{\varepsilon}\right)^{2}\left|\tilde{v}_{j}^{n+1 / 2}-A\left(\tilde{u}_{j}^{n+1 / 2}\right)\right|
$$

Using that $\mathcal{R}(u, A(u))=0$ and $\mathcal{R} \in \mathcal{C}^{1}\left(\mathbb{R}^{2}, \mathbb{R}\right)$ with $\partial_{v} \mathcal{R}(u, v) \leq \beta$, we also obtain that

$$
\Delta t\left|\mathcal{E}_{22, j}^{n}\right| \leq\left(\frac{\beta \Delta t}{\varepsilon}\right)^{2}\left|\tilde{v}_{j}^{n+1 / 2}-A\left(\tilde{u}_{j}^{n+1 / 2}\right)\right| .
$$

Therefore, from (4.1) in Proposition 4.1, we have

$$
\sum_{j \in \mathbb{Z}} \Delta x\left[\left|\mathcal{E}_{21, j}^{n}\right|+\left|\mathcal{E}_{22, j}^{n}\right|\right] \leq C \frac{\Delta t}{\varepsilon}\left(\mathrm{e}^{-\beta_{0} t^{n} / \varepsilon} \frac{\left\|\delta^{0}\right\|_{1}}{\varepsilon}+1\right) .
$$

On the other hand, we proceed to the evaluation of the terms $\mathcal{E}_{23, j}^{n}, \mathcal{E}_{24, j}^{n}$ and $\mathcal{E}_{25, j}^{n}$. First, for $s \in[0, \Delta t]$, we set

$$
\varphi_{\delta, x}(s)=\left[\mathcal{R}(u, v) \star \rho_{\delta}\right]\left(t^{n}+s, x-\sqrt{a}(\Delta t-s)\right) .
$$

Then, from (1.5) and (2.6), we know that $\left|\partial_{u} \mathcal{R}(u, v)\right| \leq \sqrt{a} \beta$ and $\left|\partial_{v} \mathcal{R}(u, v)\right| \leq \beta$, for any $(w, z) \in I\left(N_{0}, a_{0}\right)$, we obtain

$$
\begin{aligned}
\sum_{j \in \mathbb{Z}} \Delta x \Delta t\left|\mathcal{E}_{23, j}^{n}\right| \leq & \frac{1}{\varepsilon} \int_{\mathbb{T}}\left|\int_{0}^{\Delta t} \int_{0}^{s} \varphi_{\delta, x}^{\prime}(\eta) \mathrm{d} \eta \mathrm{d} s\right| \mathrm{d} x \\
\leq & C \frac{\Delta t}{\varepsilon} \int_{\mathbb{T}} \int_{t^{n}}^{t^{n+1}}\left(\left|\partial_{t} u_{\delta}\right|+\left|\partial_{x} u_{\delta}\right|\right)(t, x) \mathrm{d} t \mathrm{~d} x \\
& +C \frac{\Delta t}{\varepsilon} \int_{\mathbb{T}} \int_{t^{n}}^{t^{n+1}}\left(\left|\partial_{t} v_{\delta}\right|+\left|\partial_{x} v_{\delta}\right|\right)(t, x) \mathrm{d} t \mathrm{~d} x
\end{aligned}
$$

Thus we can use the estimates on the continuous relaxation system listed in Theorem 1.1. Indeed, since

$$
\left\{\begin{array}{l}
\partial_{t} u_{\delta}=-\partial_{x} v_{\delta} \\
\partial_{t} v_{\delta}=-a \partial_{x} u_{\delta}-\frac{1}{\varepsilon} \mathcal{R}_{\delta}(u, v)
\end{array}\right.
$$


we obtain, by applying a first order Taylor expansion of $\mathcal{R}$, the inequalities

$$
\begin{aligned}
\int_{\mathbb{T}}\left(\left|\partial_{t} u_{\delta}\right|+\left|\partial_{x} u_{\delta}\right|\right)(t, x) \mathrm{d} x & \leq T V(u(t))+T V(v(t)), \\
\int_{\mathbb{T}}\left(\left|\partial_{t} v_{\delta}\right|+\left|\partial_{x} v_{\delta}\right|\right)(t, x) \mathrm{d} x & \leq C\left(T V(u(t))+\frac{1}{\varepsilon}\|(v-A(u))(t)\|_{1}\right) .
\end{aligned}
$$

Hence, integrating over $t \in\left(t^{n}, t^{n+1}\right)$ and using (1.6) and (1.7), we get:

$$
\sum_{j \in \mathbb{Z}} \Delta x\left|\mathcal{E}_{23, j}^{n}\right| \leq C \frac{\Delta t}{\varepsilon}\left(T V\left(u\left(t^{n}\right)\right)+T V\left(v\left(t^{n}\right)\right)+\frac{\mathrm{e}^{-\beta_{0} t^{n} / \varepsilon}}{\varepsilon}\left\|\delta^{0}\right\|_{1}+1\right),
$$

where $C>0$ only depends on $\sqrt{a}$ and $\beta$.

Now we treat the term $\mathcal{E}_{24, j}^{n}$ using the smoothness properties (1.5) and (2.6) on $\mathcal{R}$, it gives

$$
\begin{aligned}
\sum_{j \in \mathbb{Z}} \Delta x\left|\mathcal{E}_{24, j}^{n}\right| & =\frac{1}{\varepsilon} \int_{\mathbb{T}}\left|\int_{\mathbb{T}}\left[\mathcal{R}(u, v)\left(t^{n}, x-y-\sqrt{a} \Delta t\right)-\mathcal{R}(u, v)\left(t^{n}, x-\sqrt{a} \Delta t\right)\right] \rho_{\delta}(y) \mathrm{d} y\right| \mathrm{d} x, \\
& \leq \frac{C}{\varepsilon} \int_{\mathbb{T}^{2}}\left[\left|u\left(t^{n}, x\right)-u\left(t^{n}, x-y\right)\right|+\left|v\left(t^{n}, x\right)-v\left(t^{n}, x-y\right)\right|\right] \rho_{\delta}(y) \mathrm{d} y \mathrm{~d} x .
\end{aligned}
$$

Thus, applying Fubini's theorem the $B V$ estimate on the exact solution (1.6) and the value of the integral of $\rho_{\delta}$, we get

$$
\sum_{j \in \mathbb{Z}} \Delta x\left|\mathcal{E}_{24, j}^{n}\right| \leq C \frac{\delta}{\varepsilon}\left[T V\left(u\left(t^{n}\right)\right)+T V\left(v\left(t^{n}\right)\right)\right] .
$$

Finally, to deal with the last term $\mathcal{E}_{25, j}^{n}$, we split it in two parts

$$
\begin{aligned}
\sum_{j \in \mathbb{Z}} \Delta x\left|\mathcal{E}_{25, j}^{n}\right| \leq & \frac{1}{\varepsilon} \int_{\mathbb{T}}\left|\mathcal{R}(u, v)\left(t^{n}, x-\sqrt{a} \Delta t\right)-\mathcal{R}\left(u_{\delta}, v_{\delta}\right)\left(t^{n}, x-\sqrt{a} \Delta t\right)\right| \mathrm{d} x \\
& +\frac{1}{\varepsilon} \sum_{j \in \mathbb{Z}} \int_{C_{j}}\left|\mathcal{R}\left(u_{\delta}, v_{\delta}\right)\left(t^{n}, x-\sqrt{a} \Delta t\right)-\mathcal{R}\left(\tilde{u}_{j}^{n+1 / 2}, \tilde{v}_{j}^{n+1 / 2}\right)\right| \mathrm{d} x
\end{aligned}
$$

and treat the different terms as for $\mathcal{E}_{24, j}^{n}$, we get for the first one

$$
\int_{\mathbb{T}}\left|\mathcal{R}(u, v)\left(t^{n}, x\right)-\mathcal{R}\left(u_{\delta}, v_{\delta}\right)\left(t^{n}, x\right)\right| \mathrm{d} x \leq C \delta\left[T V\left(u\left(t^{n}\right)\right)+T V\left(v\left(t^{n}\right)\right)\right] .
$$

and for the latter one using the $B V$ estimate on the exact solution (1.6),

$$
\sum_{j \in \mathbb{Z}} \int_{C_{j}}\left|\mathcal{R}\left(u_{\delta}, v_{\delta}\right)\left(t^{n}, x-\sqrt{a} \Delta t\right)-\mathcal{R}\left(\tilde{u}_{j}^{n+1 / 2}, \tilde{v}_{j}^{n+1 / 2}\right)\right| \mathrm{d} x \leq C \Delta x\left[\left\|\partial_{x} u_{\delta}\left(t^{n}\right)\right\|_{1}+\left\|\partial_{x} v_{\delta}\left(t^{n}\right)\right\|_{1},\right] .
$$

Thus, we have

$$
\sum_{j \in \mathbb{Z}} \Delta x\left|\mathcal{E}_{25, j}^{n}\right| \leq C\left(\frac{\delta}{\varepsilon}+\frac{\Delta x}{\varepsilon}\right)\left[T V\left(u\left(t^{n}\right)\right)+T V\left(v\left(t^{n}\right)\right)\right] .
$$

Gathering (5.6)-(5.9), and finally using the uniform bound on the BV norms of $(u, v)$ given in (1.6), it yields

$$
\sum_{j \in \mathbb{Z}} \Delta x\left|\mathcal{E}_{2, j}^{n}\right| \leq C\left[\frac{\Delta t}{\varepsilon}\left(\mathrm{e}^{-\beta_{0} t^{n} / \varepsilon} \frac{\left\|\delta^{0}\right\|_{1}}{\varepsilon}+1\right)+\frac{\Delta x}{\varepsilon}+\frac{\delta}{\varepsilon}\right] .
$$


Using the same arguments we also prove that

$$
\sum_{j \in \mathbb{Z}} \Delta x\left|\mathcal{E}_{4, j}^{n}\right| \leq C\left[\frac{\Delta t}{\varepsilon}\left(\mathrm{e}^{-\beta_{0} t^{n} / \varepsilon} \frac{\left\|\delta^{0}\right\|_{1}}{\varepsilon}+1\right)+\frac{\Delta x}{\varepsilon}+\frac{\delta}{\varepsilon}\right] .
$$

\subsection{Convergence proof}

Now we perform a rigorous analysis of the numerical scheme $(2.12)-(2.14)$ when $h=(\Delta t, \Delta x)$ goes to zero. We consider the numerical solution $\left(u_{h}^{\varepsilon}, v_{h}^{\varepsilon}\right)$ to the scheme $(2.12)-(2.14)$ and $\left(u^{\varepsilon}, v^{\varepsilon}\right)$ the exact solution to $(1.1)$ and define $\left(w^{\varepsilon}, z^{\varepsilon}\right)$ using (3.1). Then we denote by

$$
\bar{w}_{j}^{n}=\frac{1}{\Delta x} \int_{C_{j}} w^{\varepsilon}\left(t^{n}, x\right) \mathrm{d} x, \quad \bar{z}_{j}^{n}=\frac{1}{\Delta x} \int_{C_{j}} z^{\varepsilon}\left(t^{n}, x\right) \mathrm{d} x
$$

and $\left(w_{j}^{n}, z_{j}^{n}\right)_{(j, n) \in \mathbb{Z} \times \mathbb{N}}$ the numerical solution given by (3.2)-(3.3). Thus,

$$
\begin{aligned}
\sum_{j \in \mathbb{Z}} \Delta x\left[\left|w_{j}^{n}-\bar{w}_{j}^{n}\right|+\left|z_{j}^{n}-\bar{z}_{j}^{n}\right|\right] \leq & \sum_{j \in \mathbb{Z}} \Delta x\left[\left|w_{j}^{n}-\tilde{w}_{j}^{n}\right|+\left|z_{j}^{n}-\tilde{z}_{j}^{n}\right|\right] \\
& +\sum_{j \in \mathbb{Z}} \Delta x\left[\left|\tilde{w}_{j}^{n}-\bar{w}_{j}^{n}\right|+\left|\tilde{z}_{j}^{n}-\bar{z}_{j}^{n}\right|\right],
\end{aligned}
$$

where $\left(\tilde{w}_{j}^{n}, \tilde{z}_{j}^{n}\right)_{(j, n) \in \mathbb{Z} \times \mathbb{N}}$ is given by (5.3). On the one hand, we estimate the second terms of the right hand side using the convolution properties and have

$$
\sum_{j \in \mathbb{Z}} \Delta x\left[\left|\tilde{w}_{j}^{n}-\bar{w}_{j}^{n}\right|+\left|\tilde{z}_{j}^{n}-\bar{z}_{j}^{n}\right|\right] \leq C \delta[T V(u)+T V(v)] .
$$

On the other hand, we apply the consistency error analysis to estimate the first term of the right hand side. Applying (3.6)-(3.7) established in Lemma 3.1 with $\left(\tilde{w}_{j}, \tilde{z}_{j}\right)$ and $\left(w_{j}, z_{j}\right)$, it yields

$$
\begin{aligned}
\sum_{j \in \mathbb{Z}} \Delta x\left|\tilde{w}_{j}^{n+1}-w_{j}^{n+1}\right| \leq & \sum_{j \in \mathbb{Z}} \Delta x\left|\tilde{w}_{j}^{n+1 / 2}-w_{j}^{n+1 / 2}\right|\left(1+\partial_{w} G_{\varepsilon, \Delta t}\left(w_{j}, z_{j}^{n+1 / 2}\right)\right) \\
& +\sum_{j \in \mathbb{Z}} \Delta x\left|\tilde{z}_{j}^{n+1 / 2}-z_{j}^{n+1 / 2}\right| \partial_{z} G_{\varepsilon, \Delta t}\left(\tilde{w}_{j}^{n+1 / 2}, z_{j}\right) \\
& +\sum_{j \in \mathbb{Z}} \Delta x \Delta t\left[\left|\mathcal{E}_{1, j}^{n}\right|+\left|\mathcal{E}_{2, j}^{n}\right|\right]
\end{aligned}
$$

and

$$
\begin{aligned}
\sum_{j \in \mathbb{Z}} \Delta x\left|\tilde{z}_{j}^{n+1}-z_{j}^{n+1}\right| \leq & \sum_{j \in \mathbb{Z}} \Delta x\left|\tilde{z}_{j}^{n+1 / 2}-z_{j}^{n+1 / 2}\right|\left(1-\partial_{z} G_{\varepsilon, \Delta t}\left(\tilde{w}_{j}^{n+1 / 2}, z_{j}\right)\right) \\
& -\sum_{j \in \mathbb{Z}} \Delta x\left|\tilde{w}_{j}^{n+1 / 2}-w_{j}^{n+1 / 2}\right| \partial_{w} G_{\varepsilon, \Delta t}\left(w_{j}, z_{j}^{n+1 / 2}\right) \\
& +\sum_{j \in \mathbb{Z}} \Delta x \Delta t\left[\left|\mathcal{E}_{3, j}^{n}\right|+\left|\mathcal{E}_{4, j}^{n}\right|\right] .
\end{aligned}
$$

Summing the two inequalities and using that the scheme (3.3) is TVD, we get the following inequality

$$
\begin{aligned}
\sum_{j \in \mathbb{Z}} \Delta x\left[\left|\tilde{z}_{j}^{n+1}-z_{j}^{n+1}\right|+\left|\tilde{w}_{j}^{n+1}-w_{j}^{n+1}\right|\right] \leq & \sum_{j \in \mathbb{Z}} \Delta x\left[\left|\tilde{z}_{j}^{n}-z_{j}^{n}\right|+\left|\tilde{w}_{j}^{n}-w_{j}^{n}\right|\right] \\
& +\sum_{j \in \mathbb{Z}} \Delta x \Delta t\left[\left|\mathcal{E}_{1, j}^{n}\right|+\left|\mathcal{E}_{2, j}^{n}\right|+\left|\mathcal{E}_{3, j}^{n}\right|+\left|\mathcal{E}_{4, j}^{n}\right|\right] .
\end{aligned}
$$


Therefore,

$$
\begin{aligned}
\sum_{j \in \mathbb{Z}} \Delta x\left[\left|\tilde{z}_{j}^{n+1}-z_{j}^{n+1}\right|+\left|\tilde{w}_{j}^{n+1}-w_{j}^{n+1}\right|\right] \leq & \sum_{j \in \mathbb{Z}} \Delta x\left[\left|\tilde{z}_{j}^{0}-z_{j}^{0}\right|+\left|\tilde{w}_{j}^{0}-w_{j}^{0}\right|\right] \\
& +\sum_{k=0}^{n} \sum_{j \in \mathbb{Z}} \Delta x \Delta t\left[\left|\mathcal{E}_{1, j}^{k}\right|+\left|\mathcal{E}_{2, j}^{k}\right|+\left|\mathcal{E}_{3, j}^{k}\right|+\left|\mathcal{E}_{4, j}^{k}\right|\right] .
\end{aligned}
$$

Finally the consistency error analysis performed in Propositions 5.1 and 5.2 yields, taking $\delta=\Delta x^{1 / 2}$

$$
\begin{aligned}
\sum_{j \in \mathbb{Z}} \Delta x\left[\left|\tilde{z}_{j}^{n+1}-z_{j}^{n+1}\right|+\left|\tilde{w}_{j}^{n+1}-w_{j}^{n+1}\right|\right] \leq & \sum_{j \in \mathbb{Z}} \Delta x\left[\left|\tilde{z}_{j}^{0}-z_{j}^{0}\right|+\left|\tilde{w}_{j}^{0}-w_{j}^{0}\right|\right] \\
& +\frac{C}{\varepsilon}\left(\Delta t(\Delta t+\varepsilon)\left(\frac{\left\|\delta^{0}\right\|_{1}}{\varepsilon}+1\right)\right. \\
& \left.+t^{n}\left[\Delta x+\varepsilon \Delta x^{1 / 2}+\Delta x^{1 / 2}\right]\right) .
\end{aligned}
$$

Gathering (5.10) and (5.11), the right hand side converges to zero when $h=(\Delta t, \Delta x)$ goes to zero, which proves the convergence of the numerical solution $(2.12)-(2.14)$ to the exact solution to (1.1).

Therefore, from the smoothness of the exact solution and the initial data $\left(u_{0}, v_{0}\right)$, it proves that for any discretization parameter $h$, for all $\varepsilon>0$ :

$$
\int_{\mathbb{T}}\left|u_{h}^{\varepsilon}(t, x)-u^{\varepsilon}(t, x)\right|+\left|v_{h}^{\varepsilon}(t, x)-v^{\varepsilon}(t, x)\right| \mathrm{d} x \leq \frac{C}{\varepsilon}\left(\Delta t\left(\frac{\left\|\delta^{0}\right\|_{1}}{\varepsilon}+1\right)+\Delta x^{1 / 2}\right) .
$$

\section{Numerical Simulations}

This section is devoted to the numerical simulation of (1.1). We consider a nonlinear source term $\mathcal{R}(u, v)$ given by

$$
\mathcal{R}(u, v)=\frac{v-u^{2}}{1+u^{2}+v^{2}} .
$$

On the one hand we compute approximations for different meshes in space and time in order to evaluate the order of accuracy of the numerical scheme for different regimes corresponding to small and large values of the relaxation parameter $\varepsilon>0$. To this aim, we define an estimate of the numerical error by

$$
\mathcal{E}_{1}(h)=\left\|u_{h}^{\varepsilon}-u_{2 h}^{\varepsilon}\right\|_{1}+\left\|v_{h}^{\varepsilon}-v_{2 h}^{\varepsilon}\right\|_{1},
$$

where $h=(\Delta x, \Delta t)$ is the discretization parameter and $\|\cdot\|_{1}$ is the discrete $L^{1}$ norm.

On the other hand, we compare our numerical approximation with ones obtained by two other schemes. The first one is based on a splitting scheme, where the transport part is treated by (2.12)-(2.13), whereas the source term is approximated by

$$
\left\{\begin{array}{l}
u_{j}^{n+1}=u_{j}^{n+1 / 2}, \\
v_{j}^{n+1}=v_{j}^{n+1 / 2}\left(1+\frac{\beta \Delta t}{\varepsilon}\right) \mathrm{e}^{-\beta \Delta t / \varepsilon}-\frac{\Delta t}{\varepsilon} \mathrm{e}^{-\beta \Delta t / \varepsilon} \mathcal{R}\left(u_{j}^{n+1 / 2}, v_{j}^{n+1 / 2}\right) .
\end{array}\right.
$$

This scheme is uniformly stable with respect to $\varepsilon$, but it is not asymptotic preserving since $v^{n+1}$ does not converge to $A\left(u^{n+1}\right)$ when $\varepsilon$ goes to zero. 


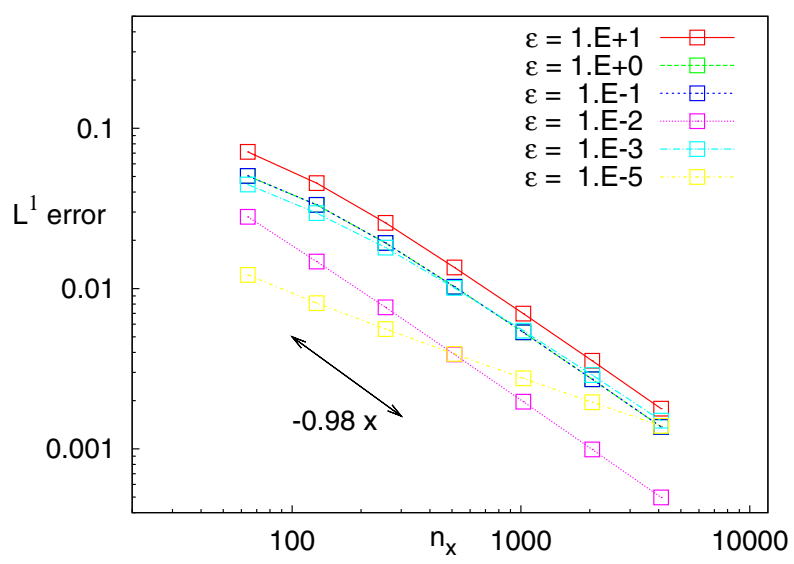

FiguRE 1. Test with smooth initial data (6.3): $L^{1}$ error for different regimes from $\varepsilon=10^{-5}$ to $\varepsilon=10$.

The second one couples the transport part (2.12)-(2.13) with a fully implicit scheme for the relaxation

$$
\left\{\begin{array}{l}
u_{j}^{n+1}=u_{j}^{n+1 / 2}, \\
v_{j}^{n+1}=v_{j}^{n+1 / 2}-\frac{\Delta t}{\varepsilon} \mathcal{R}\left(u_{j}^{n+1}, v_{j}^{n+1}\right) .
\end{array}\right.
$$

This scheme is uniformly stable with respect to $\varepsilon$ and asymptotic preserving, but it requires an additional step for the numerical resolution of the nonlinear problem (using a Newton algorithm). Note that the nonlinear problem (6.2) may have several solutions (polynomial equation of order three for $\varepsilon>0$ ) and then the convergence of the Newton or fixed point algorithm may be an open issue.

\subsection{Test with a smooth initial datum}

First we choose a smooth initial datum given by

$$
u_{0}(x)=\sin (\pi x), \quad v_{0}(x)=0, \quad \forall x \in(-1,1)
$$

and $v_{0}=0$. In Figure 1, we present the curves corresponding to the order of accuracy with respect to $h$, computed from $\mathcal{E}_{1}(h)$ and observe that the order is relatively close to one when the solution is smooth. We also observe that when $\varepsilon$ goes to zero the numerical error becomes smaller and smaller, which illustrates perfectly the uniform accuracy of the scheme for different regimes.

Then in Figures 2, 3 and 4, we propose a comparison of the numerical solutions obtained with $n_{x}=400$ obtained with our scheme (2.14), the scheme (6.1) is based on a linear penalization proposed in [12] and a fully implicit scheme (6.2) for the relaxation term. For these schemes the numerical solution is stable with respect to $\varepsilon$ and the time step $\Delta t$ is only chosen according to the CFL condition (2.7), but not with respect to $\varepsilon$, that is $\Delta t=0.003$. The numerical solutions are compared with a reference solution obtained with a fine mesh $n_{x}=10^{4}$ and $\Delta t=10^{-4}$. We observe that all the schemes give accurate results when $\varepsilon \ll 0.1$. However, the scheme (6.1) is not asymptotic preserving and does not give consistent results when $\varepsilon \rightarrow 0$ and $\Delta t$ is fixed (see Figs. 3 and 4 ). 


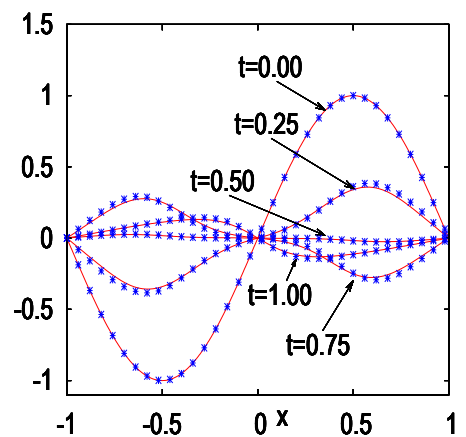

(1)

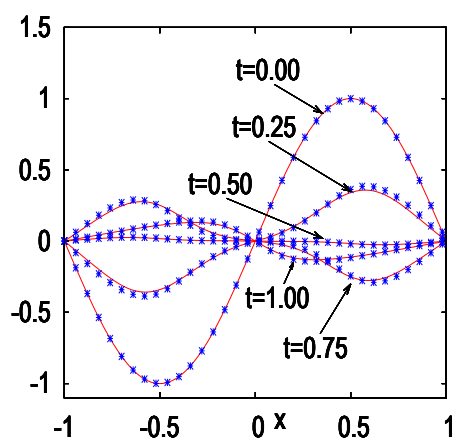

$(2)$

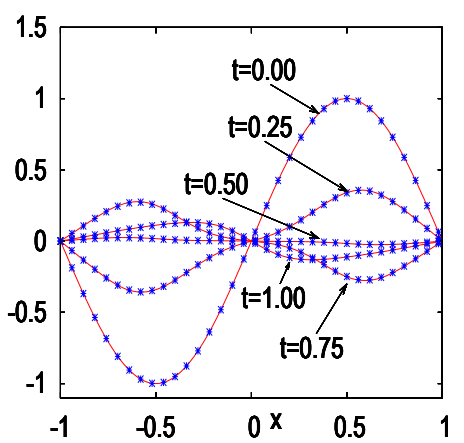

(3)

Figure 2. Test with smooth initial data (6.3): cross (x) numerical solution obtained with $n_{x}=400$ and line (-) reference solution with $n_{x}=10^{4}$. Numerical solution obtained from (1) AP scheme (2.14) (2) linear penlization (6.1) (3) fully implicit scheme (6.2) for $\varepsilon=0.1$.

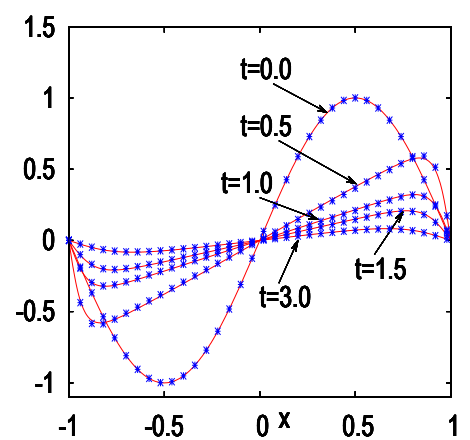

(1)

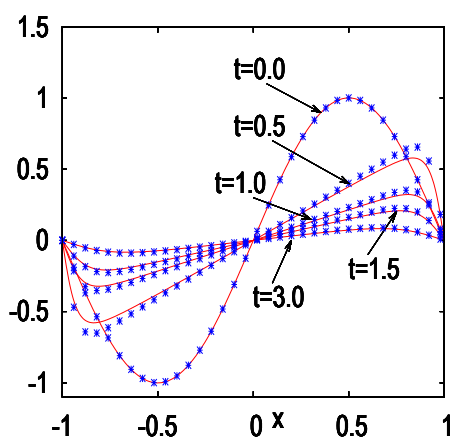

(2)

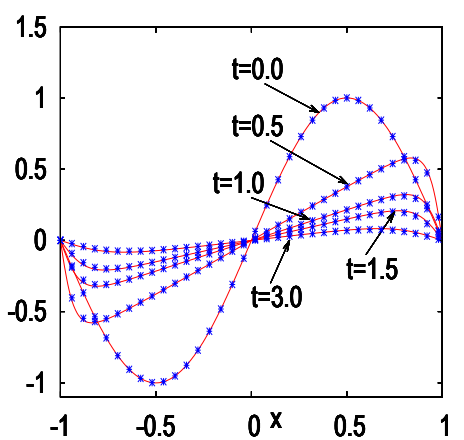

(3)

Figure 3. Test with smooth initial data (6.3): cross (x) numerical solution obtained with $n_{x}=400$ and line (-) reference solution with $n_{x}=10^{4}$. Numerical solution obtained from (1) AP scheme (2.14) (2) linear penalization (6.1) (3) fully implicit scheme (6.2) for $\varepsilon=0.01$.

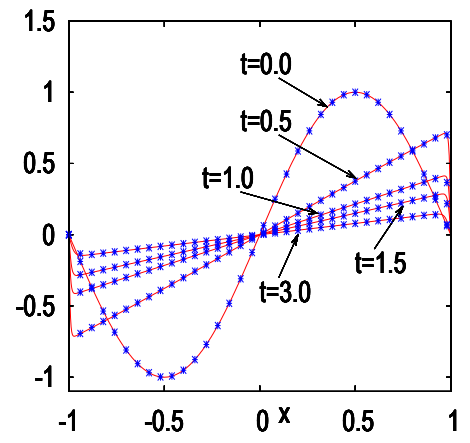

(1)

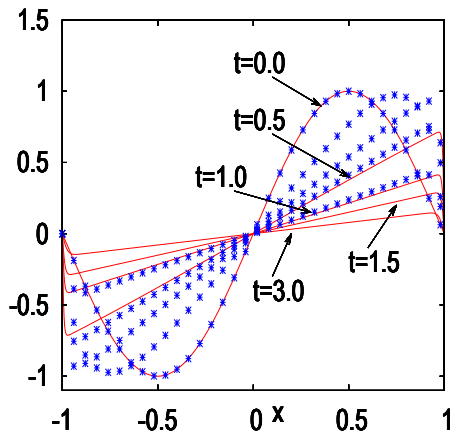

(2)

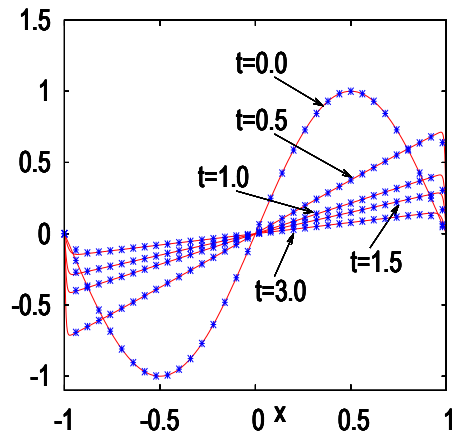

(3)

Figure 4. Test with smooth initial data (6.3): cross (x) numerical solution obtained with $n_{x}=400$ and line (-) reference solution with $n_{x}=10^{4}$. Numerical solution obtained from (1) AP scheme (2.14) (2) linear penalization (6.1) (3) fully implicit scheme (6.2) for $\varepsilon=0.001$. 


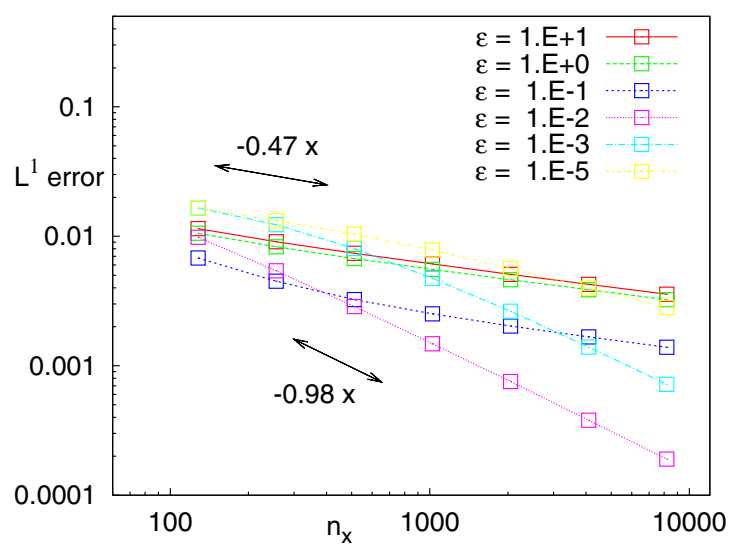

FiguRE 5. Test with a discontinuous initial datum (6.4) $L^{1}$ error for different regimes from $\varepsilon=10^{-5}$ to $\varepsilon=10$.

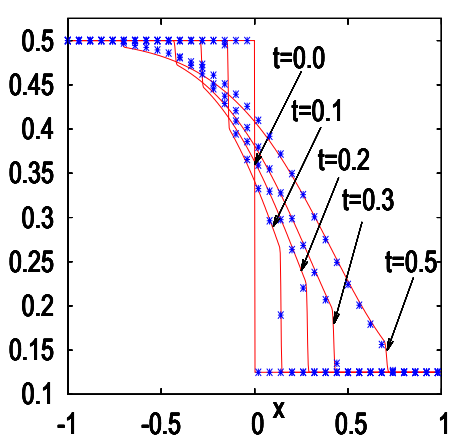

(1)

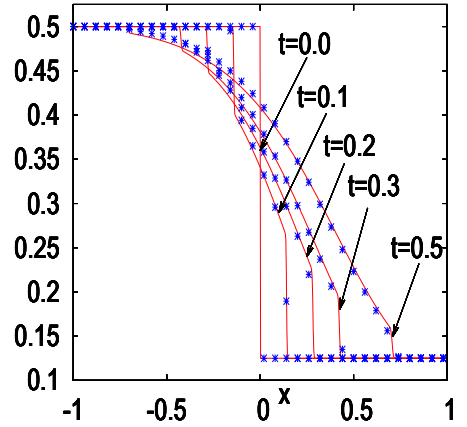

$(2)$

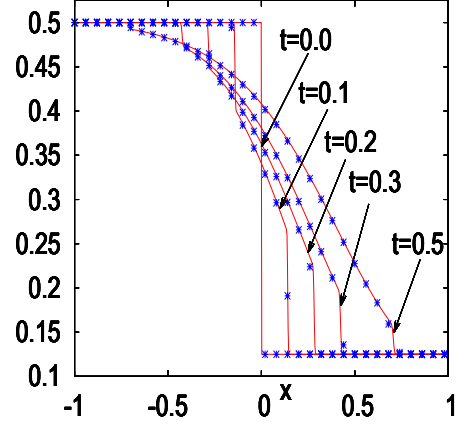

(3)

Figure 6. Test with a discontinuous initial datum (6.4): cross (x) numerical solution obtained with $n_{x}=400$ and line (-) reference solution with $n_{x}=10^{4}$. Numerical solution obtained from (1) AP scheme (2.14) (2) linear penalization (6.1) (3) fully implicit scheme (6.2) for $\varepsilon=0.1$.

\subsection{Test with a discontinuous initial datum}

Now we choose a discontinuous initial datum

$$
u_{0}(x)= \begin{cases}0.5 & \text { if }-1 \leq x \leq 0 \\ 0.125 & \text { if } 0 \leq x \leq 1\end{cases}
$$

and $v_{0}=0$.

In Figure 5, we present the curves corresponding to the order of accuracy with respect to $h$, we observe now that the order of accuracy decreases to $1 / 2$, which is coherent with the convergence analysis we performed in this paper. Moreover, the numerical error is not much affected by the variations the relaxation parameter $\varepsilon$.

Finally in Figures 6, 7 and 8, we present a comparison of the different approximations in various regimes. Our AP scheme (2.14) gives accurate results uniformly with respect to $\varepsilon$ with a computational cost of an explicit scheme even when $\Delta t$ is large compared with $\varepsilon$. 


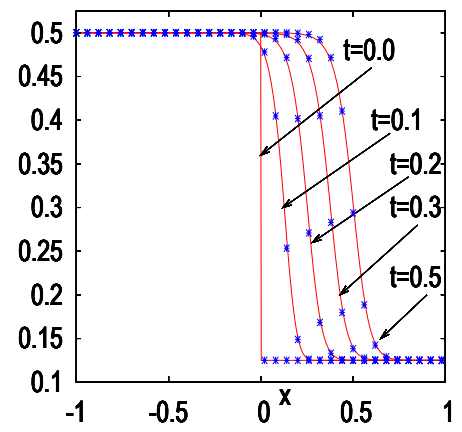

(1)

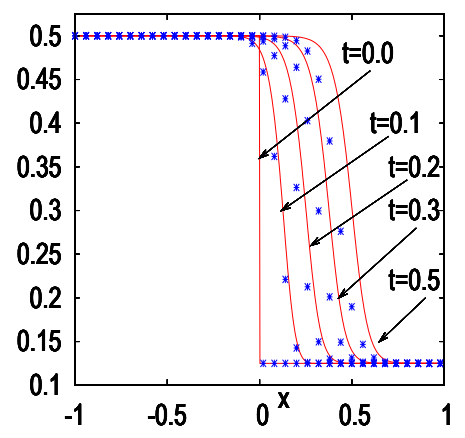

(2)

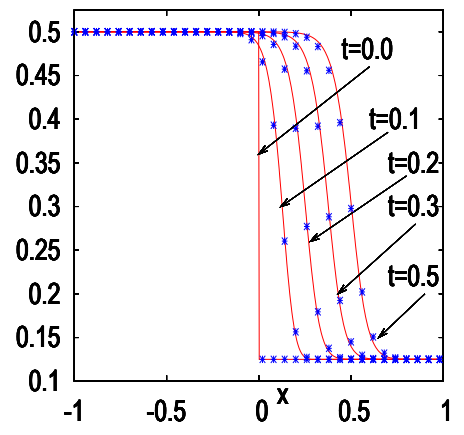

(3)

Figure 7. Test with a discontinuous initial datum (6.4): cross (x) numerical solution obtained with $n_{x}=400$ and line (-) reference solution with $n_{x}=10^{4}$. Numerical solution obtained from (1) AP scheme (2.14) (2) linear penalization (6.1) (3) fully implicit scheme (6.2) for $\varepsilon=0.01$.

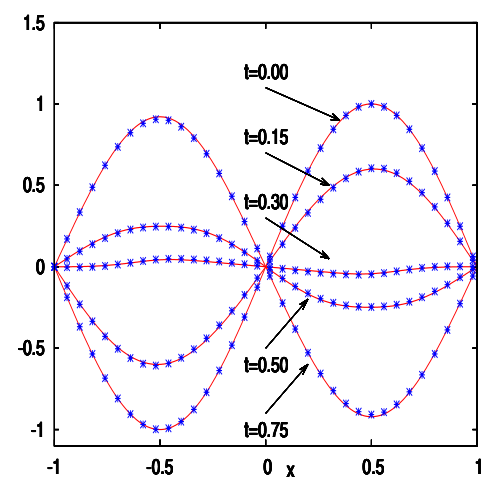

(1)

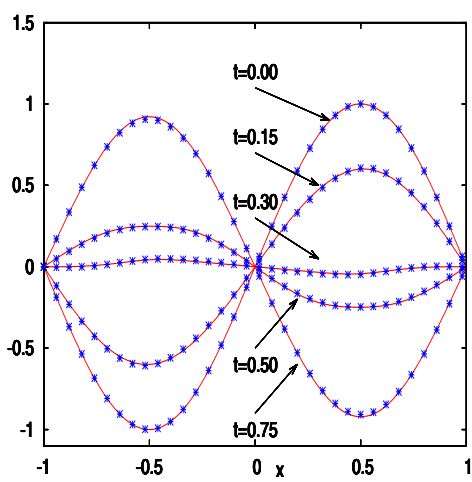

$(2)$

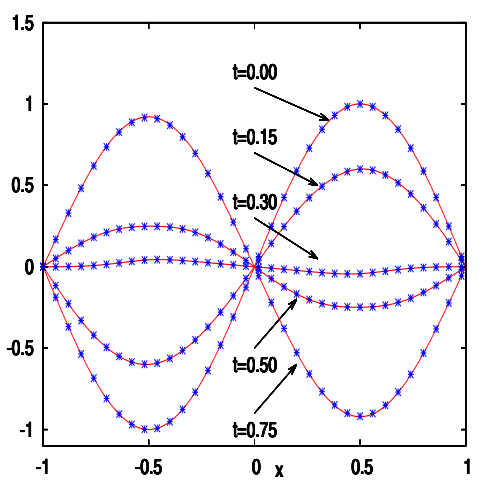

(3)

Figure 8. Test with a discontinuous initial datum (6.4): cross (x) numerical solution obtained with $n_{x}=400$ and line (-) reference solution with $n_{x}=10^{4}$. Numerical solution obtained from (1) AP scheme (2.14) (2) linear penalization (6.1) (3) fully implicit scheme (6.2) for $\varepsilon=0.001$.

\section{Conclusion}

In this paper we proposed a rigorous convergence proof of an asymptotic preserving numerical scheme applied to a system of transport equations with a nonlinear and stiff source term, for which the asymptotic limit is given by a conservation laws. We have proved the convergence of the approximate solution $\left(u_{h}^{\varepsilon}, v_{h}^{\varepsilon}\right)$ to a nonlinear relaxation system, where $\varepsilon>0$ is a physical parameter and $h$ represents the discretization parameter. Uniform convergence with respect to $\varepsilon$ and $h$ is proved and error estimates are also obtained allowing to justify rigorously the efficiency of such approach for multi-scale problems.

Note that this numerical approximation can be applied to other scaling like the "diffusive scaling" containing scales of order $1, \varepsilon$ and $\varepsilon^{2}$ [15] and more recently [4]. Our decomposition technique of the nonlinear source term together with the scheme proposed [15] leads to a robust asymptotic preserving, which can be applied to various models as Boltzmann equation for semi-conductors [8] and kinetic models for chemotaxis [4]. A rigorous convergence analysis should also work for such problems since this scaling leads to convection-diffusion models, which is mathematically simpler than nonlinear hyperbolic conservation laws. 


\section{REFERENCES}

[1] D. Aregba-Driollet and R. Natalini, Convergence of relaxation schemes for conservation laws. Appl. Anal. 1-2 (1996) 163-193.

[2] D. Aregba-Driollet and R. Natalini, Discrete kinetic schemes for multidimensional systems of conservation laws. SIAM J. Numer. Anal. 37 (2000) 1973-2004.

[3] S. Bianchini, B. Hanouzet and R. Natalini, Asymptotic behavior of smooth solutions for partially dissipative hyperbolic systems with a convex entropy. Commun. Pure Appl. Math. 60 (2007) 1559-1622.

[4] J.A. Carrillo, B. Yan, An Asymptotic Preserving Scheme for the Diffusive Limit of Kinetic systems for Chemotaxis. Preprint.

[5] A. Chalabi, Convergence of relaxation schemes for hyperbolic conservation laws with stiff source terms. Math. Comput. 68 (1999) 955-970.

[6] G.Q. Chen, T.P. Liu and C.D. Levermore, Hyperbolic conservation laws with stiff relaxation terms and entropy. Commun. Pure Appl. Math. 47 (1994) 787-830.

[7] P. Degond, J.-G. Liu and M-H Vignal, Analysis of an asymptotic preserving scheme for the Euler-Poisson system in the quasineutral limit. SIAM J. Numer. Anal. 46 (2008) 1298-1322.

[8] S. Deng, Asymptotic Preserving Schemes for Semiconductor Boltzmann Equation in the Diffusive Regime. CiCp (2012).

[9] G. Dimarco and L. Pareschi, Exponential Runge-Kutta methods for stiff kinetic equations. To appear. SIAM J. Numer. Anal. 49 (2011) 2057-2077.

[10] F. Filbet and S. Jin, A class of asymptotic preserving schemes for kinetic equations and related problems with stiff sources. J. Comput. Phys. 229 (2010).

[11] F. Filbet and S. Jin, An asymptotic preserving scheme for the ES-BGK model for he Boltzmann equation. J. Sci. Comput. $46(2011)$

[12] E. Gabetta, L. Pareschi and G. Toscani, Relaxation schemes for nonlinear kinetic equations. SIAM J. Numer. Anal. 34 (1997) $2168-2194$

[13] F. Golse, S. Jin and C.D. Levermore, The Convergence of Numerical Transfer Schemes in Diffusive Regimes I: The DiscreteOrdinate Method. SIAM J. Numer. Anal. 36 (1999) 1333-1369.

[14] L. Gosse and G. Toscani, Space localization and well-balanced schemes for discrete kinetic models in diffusive regimes. SIAM J. Numer. Anal. 41 (2003) 641-658

[15] S. Jin, L. Pareschi and G. Toscani, Diffusive Relaxation Schemes for Discrete-Velocity Kinetic Equations. SIAM J. Numer. Anal. 35 (1998) 2405-2439.

[16] S. Jin, Efficient asymptotic-preserving (AP) schemes for some multiscale kinetic equations. SIAM J. Sci. Comput. 21 (1999) $441-454$.

[17] A. Kurganov and E. Tadmor, Stiff systems of hyperbolic conservation laws: convergence and error estimates. SIAM J. Math. Anal. 28 (1997) 1446-1456.

[18] T.P. Liu, Hyperbolic conservation laws with relaxation. Commun. Math. Phys. 1 (1987) 153-175.

[19] G. Naldi and L. Pareschi, Numerical schemes for hyperbolic systems of conservation laws with stiff diffusive relaxation. SIAM J. Numer. Anal. 37 (2000) 1246-1270.

[20] R. Natalini, Convergence to equilibrium for the relaxation approximations of conservation laws. Commun. Pure Appl. Math. 8 (1996) 795-823.

[21] E. Tadmor and T. Tang, Pointwise error estimates for scalar conservation laws with piecewise smooth solutions. SIAM J. Numer. Anal. 36 (1999) 1739-1758.

[22] E. Tadmor and T. Tang, Pointwise error estimates for relaxation approximations to conservation laws. SIAM J. Math. Anal. 32 (2000) 870-886.

[23] T. Tang and J. Wang, Convergence of MUSCL relaxing schemes to the relaxed schemes of conservation laws with stiff source terms. J. Sci. Comput. 15 (2000) 173-195. 